Review

\title{
A Review of Heating and Temperature Control in Microfluidic Systems: Techniques and Applications
}

\author{
Vincent Miralles $^{1, \dagger}$, Axel Huerre ${ }^{1, \dagger}$, Florent Malloggi ${ }^{2}$ and Marie-Caroline Jullien ${ }^{1, *}$ \\ 1 Gulliver CNRS ESPCI, UMR7083, MMN, 10 rue Vauquelin, 75005 Paris, France; \\ E-Mails: vincent.miralles@ espci.fr (V.M.); axel.huerre@espci.fr (A.H.) \\ 2 SIS2M-LIONS CEA CNRS, UMR 3299, CEA Saclay, 91191 Gif-sur-Yvette, France; \\ E-Mail: florent.malloggi@cea.fr \\ $\dagger$ These authors contributed equally to this work. \\ * Author to whom correspondence should be addressed; E-Mail: marie-caroline.jullien@espci.fr; \\ Tel.: +33-1-40-79-51-61; Fax: +33-1-40-79-51-57.
}

Received: 23 November 2012; in revised form: 19 December 2012 / Accepted: 4 January 2013 / Published: 15 January 2013

\begin{abstract}
This review presents an overview of the different techniques developed over the last decade to regulate the temperature within microfluidic systems. A variety of different approaches has been adopted, from external heating sources to Joule heating, microwaves or the use of lasers to cite just a few examples. The scope of the technical solutions developed to date is impressive and encompasses for instance temperature ramp rates ranging from 0.1 to $2,000{ }^{\circ} \mathrm{C} / \mathrm{s}$ leading to homogeneous temperatures from $-3{ }^{\circ} \mathrm{C}$ to $120{ }^{\circ} \mathrm{C}$, and constant gradients from 6 to $40{ }^{\circ} \mathrm{C} / \mathrm{mm}$ with a fair degree of accuracy. We also examine some recent strategies developed for applications such as digital microfluidics, where integration of a heating source to generate a temperature gradient offers control of a key parameter, without necessarily requiring great accuracy. Conversely, Temperature Gradient Focusing requires high accuracy in order to control both the concentration and separation of charged species. In addition, the Polymerase Chain Reaction requires both accuracy (homogeneous temperature) and integration to carry out demanding heating cycles. The spectrum of applications requiring temperature regulation is growing rapidly with increasingly important implications for the physical, chemical and biotechnological sectors, depending on the relevant heating technique.
\end{abstract}


Keywords: heating; temperature; microfluidics

\section{Introduction}

The development of lab-on-a-chip requires the integration of multiple functions within a compact platform, which is readily transportable and can deliver rapid data output. One such functionality is the control of temperature, either in terms of profile (homogeneous or gradient) or in terms of the accessible range, and in both cases with the greatest accuracy possible. Indeed, the regulation of temperature is a critical parameter in managing many physical, chemical and biological applications. Prominent examples of applications requiring tight temperature control are Polymerase Chain Reaction (PCR) [1-15], Temperature Gradient Focusing for Electrophoresis (TGF) [16,17], digital microfluidics [18-31], mixing [32-34], and protein crystallization [35]. The scope of this article is to provide a comprehensive applications-based overview of heating techniques reported in the literature during the last decade. The vast majority of studies, involving heating/cooling technologies exploit external approaches such as the use of macroscopic Peltier or pre-heated liquids [1-5,36-40] flowing through the microsystem. These technologies facilitate both homogeneous temperature regulation within the whole microsystem, and linear temperature profiles often with a high degree of accuracy; however the control is not integrated and may thus limit the potential applications. In addition to the integration of micro-Peltier components, other integrated technologies have been developed using: Joule heating [15,20,21,28-30,41-46], microwaves [47-64], endothermal chemical reactions [65-66], and integrated wires and lasers [67-70].

The review is organized as follows:

The first sections focus on the different techniques reported to date. These techniques are described along with the corresponding specifications if explicitly stated in the original report (see Table 1). Techniques are classified according to their level of integration:

* Section 2 covers external heating methods, i.e., by means of commercial heaters with some degree of integration, but entirely external approaches (such as using hot-plates) are not considered here,

* Section 3 using integrated heaters within the microsystem, and,

* Section 4 heating techniques exploiting electromagnetic radiations, i.e., the liquid is directly heated in the bulk material.

* In Section 5, we introduce a range of applications that illustrate the efficacy of different heating techniques. Throughout the paper we refer to these applications, the reader not familiar with the applications is invited to refer to this section. The spectrum of applications is wide-ranging and rapidly growing; and it is not our intention to detail all the applications that may benefit from thermal control but rather to give an overview of the variety of approaches that have been adapted for different applications. 
Table 1. Summary of the specifications related to the techniques discussed in the review.

\begin{tabular}{|c|c|c|c|c|c|c|c|c|c|c|c|}
\hline \multicolumn{5}{|c|}{ Heating method } & \multirow{3}{*}{$\begin{array}{c}\text { Level of } \\
\text { integration }\end{array}$} & \multirow{3}{*}{$\begin{array}{c}\text { Range of } \\
\text { temperature } \\
\left({ }^{\circ} \mathrm{C}\right)\end{array}$} & \multicolumn{4}{|c|}{ Spatial distribution } & \multirow{3}{*}{$\begin{array}{c}\text { Power } \\
\text { needed } \\
(\mathbf{m W})\end{array}$} \\
\hline \multirow{2}{*}{$\begin{array}{l}\text { Pre-heated } \\
\text { liquids }\end{array}$} & \multicolumn{2}{|c|}{ Joule heating } & \multirow[b]{2}{*}{ Microwaves } & \multirow{2}{*}{$\begin{array}{l}\text { Chemical } \\
\text { reactions }\end{array}$} & & & \multicolumn{2}{|c|}{ Constant T } & \multicolumn{2}{|c|}{ Gradient T } & \\
\hline & $\begin{array}{c}\text { homogeneous } \\
\mathbf{T} \\
\end{array}$ & $\begin{array}{c}\text { gradient } \\
\mathbf{T} \\
\end{array}$ & & & & & $\begin{array}{c}\text { Ramp rate } \\
\left({ }^{\circ} \mathrm{C} / \mathrm{s}\right)\end{array}$ & $\begin{array}{c}\text { Accuracy } \\
\left( \pm{ }^{\circ} \mathbf{C}\right)\end{array}$ & $\begin{array}{c}\text { Maximum value } \\
\left({ }^{\circ} \mathrm{C} / \mathrm{mm}\right)\end{array}$ & $\begin{array}{c}\text { Response } \\
\text { time }\end{array}$ & \\
\hline $\begin{array}{c}{[36]} \\
{[37]} \\
{[38]} \\
{[46]} \\
{[16]} \\
{[40]} \\
{[2]}\end{array}$ & $\begin{array}{l} \\
\\
\\
\\
{[67]} \\
{[41]} \\
{[44]} \\
{[42]} \\
{[15]} \\
{[13]} \\
{[29]} \\
{[45]}\end{array}$ & 列 & $\begin{array}{l} \\
\\
\\
\\
\\
\\
\\
{[59]} \\
{[56]} \\
{[64]} \\
{[55]}\end{array}$ & [65] & $\begin{array}{c}+ \\
+ \\
++ \\
++ \\
++ \\
+++ \\
+++ \\
++ \\
++ \\
+++ \\
++ \\
+++ \\
+++ \\
+++ \\
+++ \\
+++ \\
+++ \\
++ \\
+ \\
+ \\
+ \\
+++ \\
+++ \\
+++\end{array}$ & $\begin{array}{c}5-45 \\
5-45 \\
10-80 \\
10-80 \\
5-60 \\
-3-120 \\
22-95 \\
20-96 \\
20-130 \\
50-100 \\
25-130 \\
25-96 \\
25-96 \\
25-55 \\
25-96 \\
25-96 \\
25-96 \\
25-75 \\
25-32 \\
20-70 \\
20-96 \\
21-51 \\
31-53 \\
-3-76\end{array}$ & $\begin{array}{c}+4-3 \\
+4-3 \\
\\
\\
+106-89 \\
+100-90 \\
+20-11.5 \\
+0.1 \\
+20-10 \\
\\
+20-10 \\
+20-20 \\
\\
+20 \\
+7 \\
+65 \\
+2,000-30,000 \\
1\end{array}$ & $\begin{array}{c}0.3 \\
1 \\
\\
\\
\\
0.2 \\
0.1 \\
0.5 \\
0.2 \\
1 \\
\\
\\
0.2 \\
0.6 \\
\\
2 \\
\text { not stable } \\
0.1 \\
1.4\end{array}$ & $\begin{array}{c}5.8 \\
25 \\
13.75 \\
\end{array}$ & $\sim 3$ min & $\begin{array}{c}500 \\
300 \\
500 \\
500 \\
1,000 \\
10 \\
400 \\
500 \\
\\
1,000 \\
0\end{array}$ \\
\hline
\end{tabular}


All the reported techniques, as well as the conditions reported (heating method, level of integration, range of temperature, spatial distribution and power needed), are summarized in a table at the beginning of the paper. Generally, it seems that there is currently no consensus on any given technique that would satisfy all the requirements specified by the complete range of applications; however each of the techniques described here has successfully demonstrated the integration of temperature control for specific applications.

Note also that temperature mapping techniques are beyond the scope of the current paper, for this we refer the reader to the recent review by Gosse, Bergaud and Löw [71].

\section{External Heating}

This section describes techniques based on commercial heaters, either to heat liquids prior to being injected into the microsystem, (pre-heated liquids), or the incorporation of commercial components such as Peltier elements. Depending on the final application it is possible to generate either uniform temperature control or temperature gradients, as outlined in the next subsections.

\subsection{Homogeneous Temperature}

A number of techniques using pre-heated liquids have been reported for microfluidic devices. These methods utilize microheaters such as Peltier elements to establish either a uniform temperature or a constant gradient in a given region. Velve Casquillas and co-workers [36,37] developed a disposable polydimethylsiloxane (PDMS) based microfluidic device consisting of two Peltier stages controlling the temperature of the liquid flowing through a control channel (Figure 1(a)). The virtue of PDMS is its relatively low thermal conductivity $(0.15 \mathrm{~W} / \mathrm{mK}$ typically), which allows efficient heat transfer from the source towards the liquid (minimizing energy losses) [43]. This integrated system is capable of reversibly switching between $5{ }^{\circ} \mathrm{C}$ and $45^{\circ} \mathrm{C}$ in less than $10 \mathrm{~s}$ (Figure 1(b)). Changing the direction of the liquid flow through either a cold or hot Peltier using a syringe pump changes the temperature of cells located underneath the temperature control channel. To characterize the temperature response of the chamber, a thin platinum resistance $(50 \mathrm{~nm})$ was bonded to the microchannel block. As the electrical resistance of platinum changes nearly linearly with temperature, the authors could record the temperature inside the cell channels by measuring the resistance in the wire.

The previous example shows the potential to exploit external Peltier elements, typically by positioning these elements underneath a microchip. Maltezos and co-workers [2,3] report the use of a microfluidic thermal heat exchanger to cool a Peltier junction and demonstrate rapid heating and cooling of small volumes of solution (typically $0.4 \mu \mathrm{L}$ ). The microfluidic device is able to perform very fast cycling over a temperature range from 22 to $95{ }^{\circ} \mathrm{C}$. The introduction of four parallel Peltier junctions resulted in ramp rates of about $100{ }^{\circ} \mathrm{C} / \mathrm{s}$ for heating, and $90{ }^{\circ} \mathrm{C} / \mathrm{s}$ for cooling. In a nutshell, this simple technique represents a miniaturized PCR-on-a-chip system to amplify DNA fragments.

More sophisticated set-ups have been described by Khandurina et al. [4] who have developed a device consisting of a compact thermal cycling assembly based on Peltier elements surrounding a microchip gel electrophoresis platform for rapid PCR based analysis. The temperature ramp rates achieved are typically $20-30{ }^{\circ} \mathrm{C} / \mathrm{s}$. For amplification, the temperature steps are $94{ }^{\circ} \mathrm{C}, 50{ }^{\circ} \mathrm{C}$ and $72{ }^{\circ} \mathrm{C}$ with hold times of 30, 20, $25 \mathrm{~s}$, resulting in $\sim 1.25 \mathrm{~min} /$ cycle (Figure 2). 
Figure 1. (a) A schematic representation of the control device: temperature is set by an external Peltier element; the yeast channel is placed below the temperature control channel (b) Temperature versus time plot showing a heating rate of $4{ }^{\circ} \mathrm{C} / \mathrm{s}$. Reprinted from [37], Copyright 2010, with permission from Elsevier.

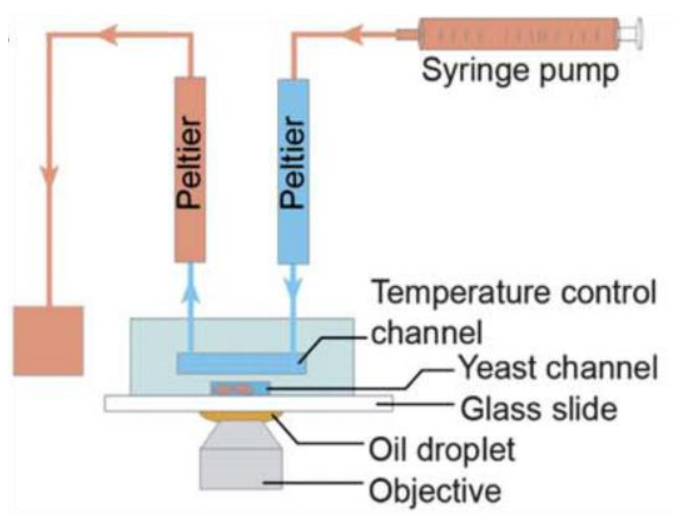

(a)

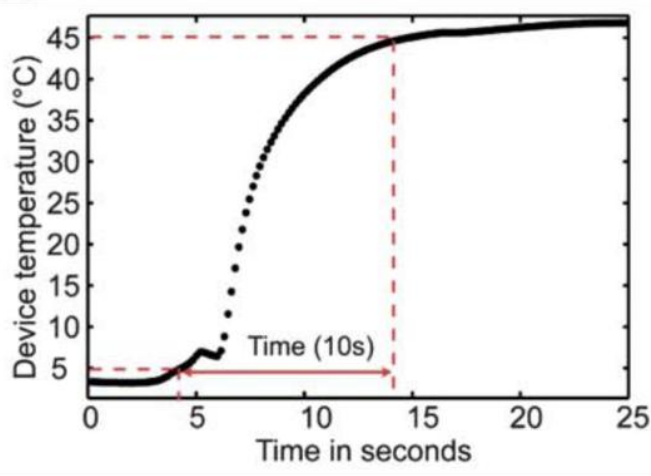

(b)

Figure 2. Temperature versus time for Polymerase Chain Reaction (PCR) amplification. The gray solid line represents the set-point temperature, the black solid line is the temperature of the bottom Peltier element and the dashed line is the reaction mix temperature. Reprinted with permission from [4]. Copyright 2000 American Chemical Society.

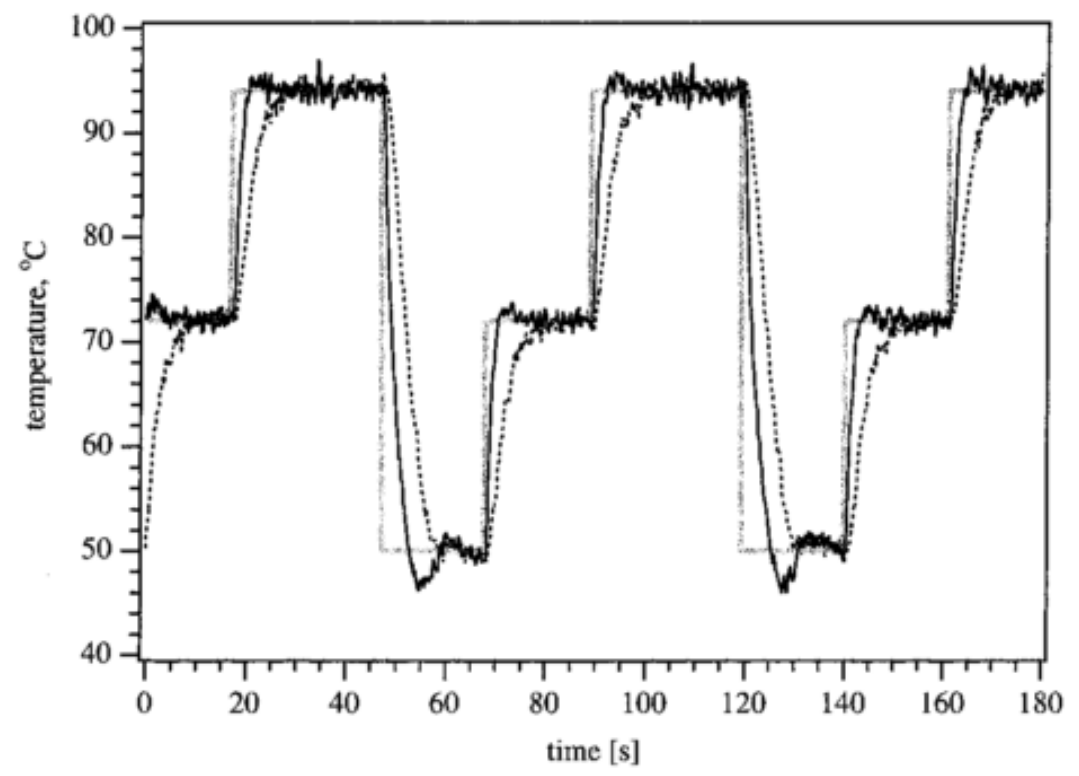

Along similar lines, Yang et al. [5] used a serpentine shaped thin $(0.75 \mathrm{~mm})$ polycarbonate PCR micro reactor and demonstrated its detection sensitivity and specificity in amplification of the E. coli K12-specific gene fragment. During thermal cycling, the PCR device is sandwiched between two Peltier elements (Figure 3). The authors performed 30 cycles in $30 \mathrm{~min}$ and were able to amplify the K12-specific gene from 10 cells in the presence of $2 \%$ blood. Peltier surface and intra-chamber temperatures are transduced by thermocouples which regulate the temperature cycles. Heating rates of $7-8{ }^{\circ} \mathrm{C} / \mathrm{s}$ and cooling rates of $5-6^{\circ} \mathrm{C} / \mathrm{s}$ can be achieved using this technique. 
Figure 3. A picture of the chip included in the heating-cooling device. Reprinted with permission from [5]. Copyright 2002, Royal Society of Chemistry.

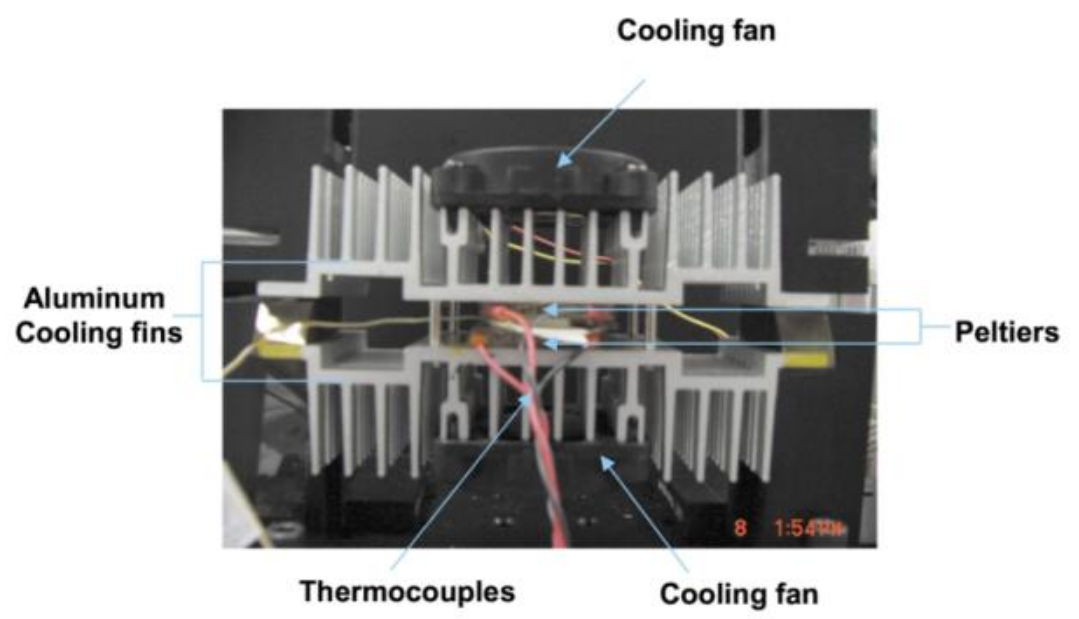

Qiu et al. [11] described a new method to perform PCR diagnostics based on plastic microfluidic reactors with relatively large volumes $(10$ to $100 \mu \mathrm{L})$. The device is a portable thermal cycler combined with a compact detector for real-time PCR, which can quantify the amount of amplified DNA during an experiment. The chip is located between the master thermoelectric element and a thermal plate. The system achieves a temperature ramp rate of approximately $4{ }^{\circ} \mathrm{C} / \mathrm{s}$ for heating and $6{ }^{\circ} \mathrm{C} / \mathrm{s}$ for cooling, and the temperature of the liquid in the reaction chamber follows the set-point temperature with an accuracy of $\pm 0.1^{\circ} \mathrm{C}$ up to a temperature of $94{ }^{\circ} \mathrm{C}$.

Maltezos et al. [2,3] integrated micro-Peltier junctions of size $0.6 \times 0.6 \times 1 \mathrm{~mm}^{3}$ into their microfluidic device in order to heat and cool nanoliter fluid volumes. These junctions generate a temperature range from $-3{ }^{\circ} \mathrm{C}$ to $120{ }^{\circ} \mathrm{C}$ with an accuracy of about $0.2{ }^{\circ} \mathrm{C}$, and good long-term stability. Temperature rates of $106^{\circ} \mathrm{C} / \mathrm{s}$ for heating and $89{ }^{\circ} \mathrm{C} / \mathrm{s}$ for cooling were achieved.

Apart from PCR applications, Liu et al. [72] developed a valving mechanism using paraffin, which undergoes solid-liquid phase transition in response to changes in temperature. As shown in Figure 4, a block of paraffin initially blocks the channel. The paraffin is melted by a heater located directly underneath the chip, and moved downstream by pressure coming from an upstream channel. Once the molten paraffin moves out of the heating zone, it begins to solidify on the wall of a wider channel. The opening of the valve is single use and facilitates transportability in a sealed system. However, the response time of devices mentioned above are of the order of 5-10 s, which is relatively high compared to other systems which bring into play pressure controlled on-off valves $[73,74]$.

Mahjoob et al. [7] introduced porous inserts with high temperature conductivity to improve heat transfer by providing a large surface area for a given volume. The system is assembled in three layers: the porous medium is located above an impermeable conductive plate and the microchip is placed underneath this plate. An optimized technique is established based on the effects of several parameters (heat exchanger geometry, conductive plate, porous matrix material used. etc.) on the temperature distribution and the power required to circulate the fluid in the heat exchanger. The heating/cooling ramp of the PCR heat exchanger is equal to $150.82{ }^{\circ} \mathrm{C} / \mathrm{s}$, which is considerably higher than results reported elsewhere in the literature. 
Figure 4. (a-b) Schematic illustrations of a close-open paraffin microvalve design. (c-e) An open-close-open microvalve design. (f) A photograph of a PCR chamber surrounded by five paraffin-based microvalves: valves 1-3 are open-close valves, and valves 4 and 5 are close-open valves. Reprinted with permission from [72]. Copyright 2004 American Chemical Society.

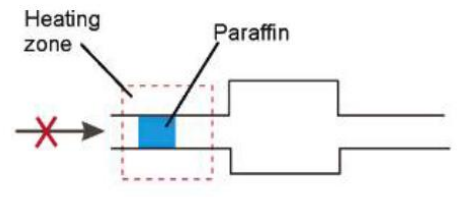

A

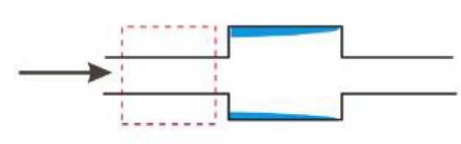

B

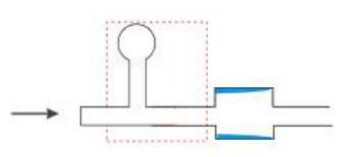

E

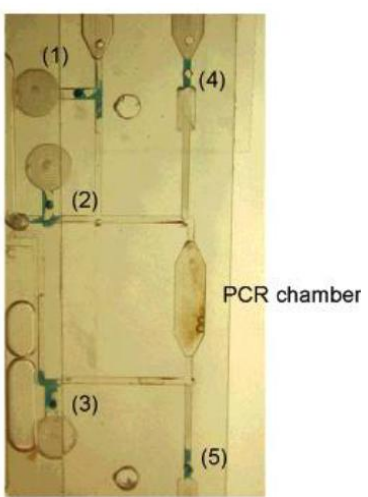

$\mathbf{F}$

\subsection{Temperature Gradient}

It is also possible to generate temperature gradients using the pre-heated liquids approach as reported by Mao et al. [38]. A linear temperature gradient is generated across dozens of parallel microfluidic channels simultaneously, located in between a hot source and a cold sink separated by a straight wall (Figure 5). The device was manufactured using soft lithographic techniques [39] and its dimensions range from $20 \times 7 \mu \mathrm{m}^{2}$ up to $250 \times 7 \mu \mathrm{m}^{2}$. The linear temperature profile of $5.8{ }^{\circ} \mathrm{C} / \mathrm{mm}$ depicted in Figure 5 was measured in a microfluidic device composed of eight parallel channels located in between the heating and cooling tubes. A thermocouple is placed at different locations giving rise to the plot presented on Figure 5.

Figure 5. A schematic of the device producing a linear temperature gradient. $\mathrm{q}_{\mathrm{x}}$ is a representation of the heat flux going from the hot source on the left to the cold one on the right. Reprinted with permission from [38]. Copyright 2002 American Chemical Society.

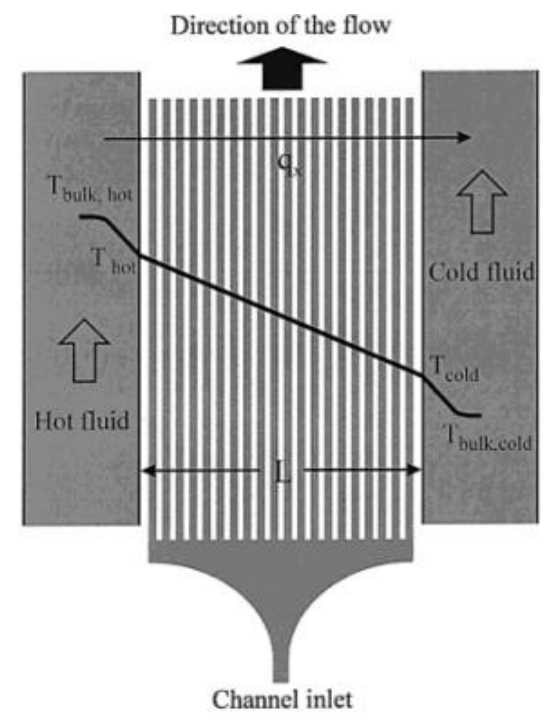


In a similar approach, Matsui and co-workers [16] integrated two Peltier elements to generate a temperature gradient, which can achieve temperature gradients of $13.75{ }^{\circ} \mathrm{C} / \mathrm{mm}$ across a $4 \mathrm{~mm}$ gap. The dimensions of the Peltier elements are $20 \mathrm{~mm}$ wide, $40 \mathrm{~mm}$ in length and $3.4 \mathrm{~mm}$ in height. The authors combine a temperature gradient, an applied electric field and a buffer with a temperature-dependent ionic strength in order to focus analytes by balancing their electrophoretic velocities against the bulk velocity of the buffer containing the analytes (TGF). In 45 s, Oregon Green 488 carboxylic acid is concentrated approximately 30 fold by applying a moderate electrical field of $70 \mathrm{~V} / \mathrm{cm}$ and a temperature gradient of $13.75^{\circ} \mathrm{C} / \mathrm{mm}$ across a $4 \mathrm{~mm}$ gap.

Finally, the generation of temperature gradients using Peltier elements can be applied to map-out solubility phase diagrams. Laval et al. [35] devised a new microfluidic chip that allows the direct and quantitative reading of two-dimensional solubility diagrams (Figure 6). Firstly, droplets containing a solute with a gradual variation of concentration are stocked on the chip. Crystallization is induced in these droplets by rapid cooling, and finally, a temperature gradient is applied to dissolve crystals in droplets at temperatures higher than their solubility temperature. As a result, they directly sample the solubility boundary between droplets with and without crystals, which gives the solubility temperatures at different concentrations (i.e., 2D-readable system: abscissa with temperature, and ordinate with concentration). The temperature field of the chip is controlled by two Peltier elements located underneath a silicon wafer which forms a chip support to optimize thermal transfers, and generates regular temperature gradients of about $0.7^{\circ} \mathrm{C} / \mathrm{mm}$ along the storage channels. This original technique is simple and cheap and could potentially be used in high throughput studies, given the small amount of reagents needed (around $250 \mu \mathrm{L}$ ).

Figure 6. (a) Design of the microfluidic device (channel width 500microns). Silicone oil is injected in inlet 1 and aqueous solutions in inlets 2 and 3. The two dotted areas indicate the positions of Peltier modules used to apply temperature gradients. The three lines of dots mark the positions of temperature measurements. (b) Example of directly reading out of a solubility diagram. The dotted line surrounding droplets containing crystals gives an estimation of the solubility limit. Reprinted with permission from [35]. Copyright 2007, Royal Society of Chemistry.
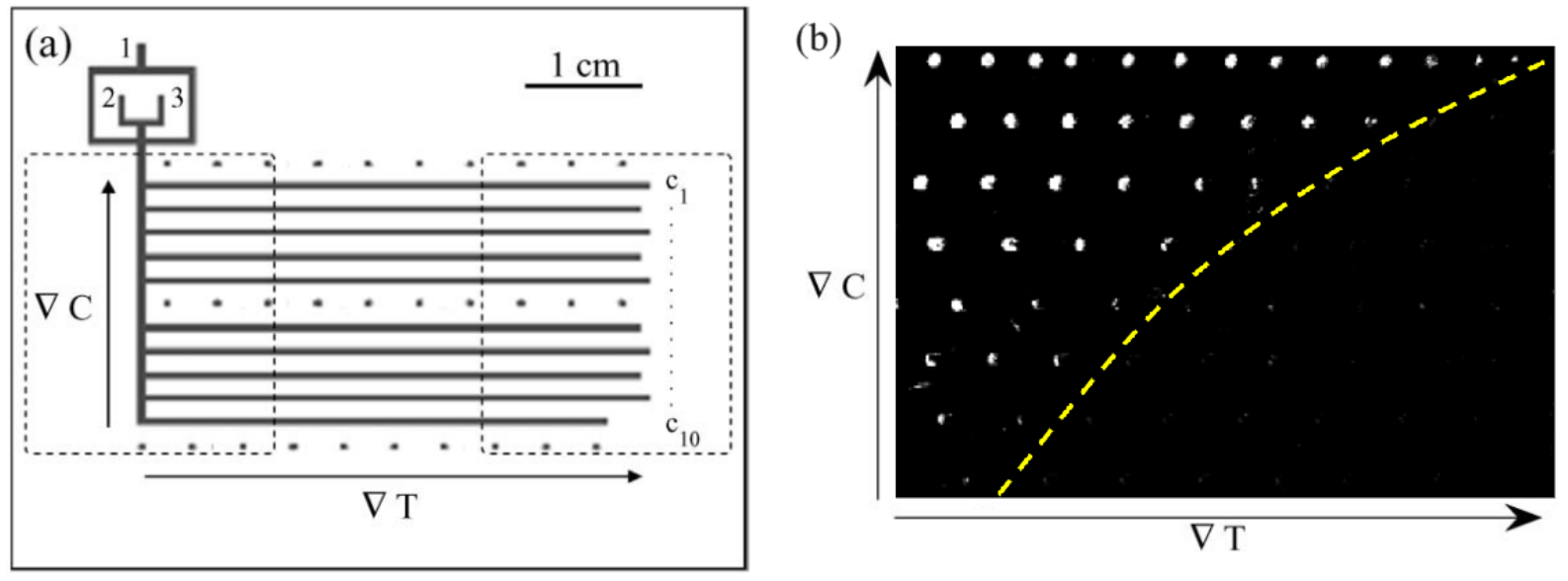

Peltier elements are widely used to create hot/cold zones, and are able to generate a spatial distribution of temperature with impressive accuracy. However, for many techniques, these elements 
are not considered as an integral part of the microfluidic chip because of their size, which is typically several millimeters. However, methods have been developed to integrate heating or cooling functionalities directly into microfluidic systems. These approaches are presented in the following sections.

\section{Integrated Heating}

We now turn to integrated techniques, from which heat diffuses from/to the integrated heating/cooling source. The first example we present derives from the use of a chemical reaction. In 2002, Guijt et al. [65] made use of endothermic and exothermic processes to locally regulate temperature in a microchannel. This method is fully integrated and cost effective with channels of typical dimensions: $54 \mu \mathrm{m}$ wide and $19 \mu \mathrm{m}$ deep. For cooling, the evaporation of acetone (Reagent 1) in the air (Reagent 2) is used as an endothermic process. For heating, the dissolution of $97 \mathrm{wt} \%$ $\mathrm{H} 2 \mathrm{SO} 4$ (Reagent 1) in water (Reagent 2) is used as an exothermic reaction. The central channel (represented in red on Figure 7) is filled with a solution of $1 \mu \mathrm{M}$ Rhodamine B in water so that the fluorescence gives a direct measurement of the temperature inside the microchannel. Note that heating experiments were conducted in glass-glass channels whereas cooling trials were carried out in PDMS-glass systems. By tuning the flow rate ratio between the two reagents, the authors demonstrate control over the intensity of the reaction and hence the temperature. This approach can achieve temperatures ranging from $-3{ }^{\circ} \mathrm{C}$ up to $76^{\circ} \mathrm{C}$ with ramps about $1{ }^{\circ} \mathrm{C} / \mathrm{s}$.

Figure 7. Two reactant channels merging into a temperature control channel, running parallel to the working channel.

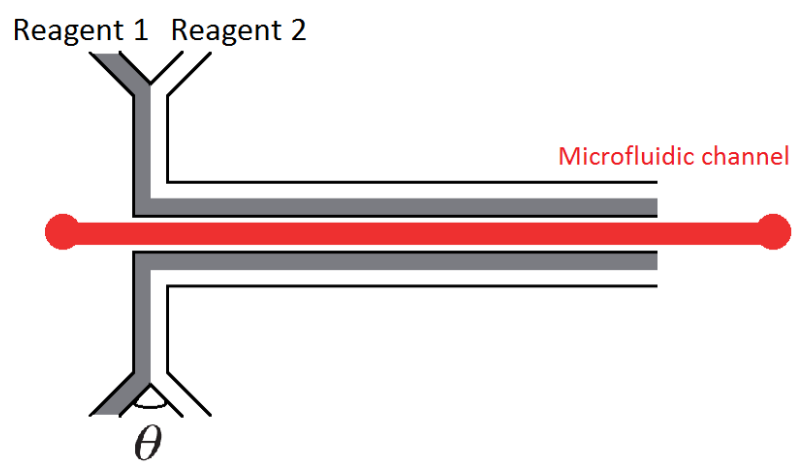

This kind of approach was optimized by Maltezos et al. in 2006 [66] for cooling. The authors compared a range of different solvents and angles ( $\theta$ in the schematic) of the Y-junction, evaporated in a $\mathrm{N}_{2}$ flux. They concluded that the most efficient solvent they tested was di-ethyl ether with an angle of $10^{\circ}$, which offers the possibility to cool down to $-20^{\circ} \mathrm{C}$ with a steady state for several minutes. This method is again cheap and clearly suited for microfluidic applications but requires further refinement of the heating control to work efficiently in PDMS channels.

The following section concerns the most widely reported technique in the literature, based on Joule heating temperature control approaches [20,21,28-30,41-46]. The technique relies on a simple physical property of conducting metals or liquids. Whichever technique is used to embed heating resistors in a microfluidic system, a linear relationship can be demonstrated between the dissipated power (given by the applied potential and the resistance of the heater) and the heat flux. A stationary temperature profile (Figure 8) can be achieved either by the addition of a heat sink, or by feedback control 
requiring the integration of a sensor (this point is critical for all techniques in which power is applied - increasing the mean temperature - as opposed to imposing a temperature). In addition, due to the small size of the heaters, the required heating power generated is in the range of $1 \mathrm{~W}$ by applying only a few Volts.

Figure 8. (a) Calibration curves: plot of the resistance $\mathrm{R}$ versus temperature $\mathrm{T}$ for the three microheaters. Reprinted from [42], Copyright 2011, with permission from Elsevier. (b) Temperature increase as a function of power supply. Reprinted from [45], with kind permission from Springer Science+Business Media.
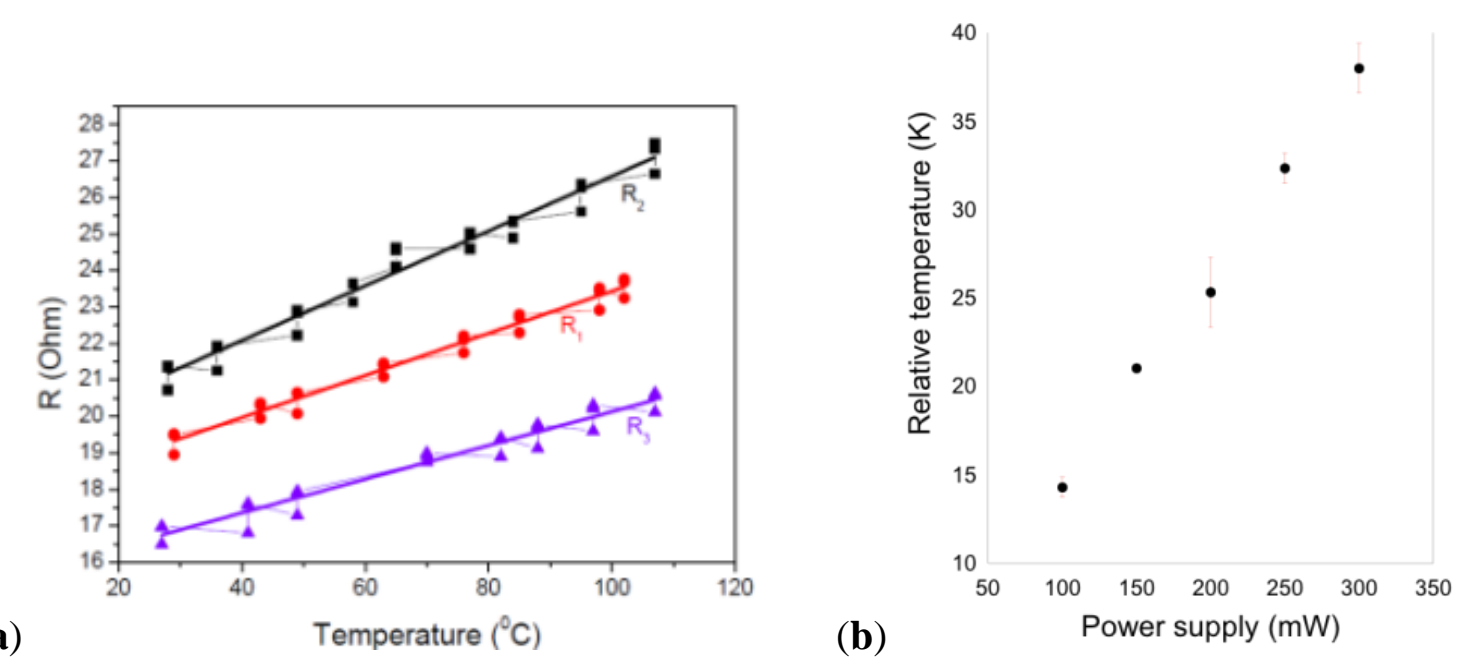

Thermal actuation of microfluidic valves by generating a heating pulse has recently been reported. Pitchaimani et al. [75] used a PDMS based microfluidic chip to control fluid flow in microchannels. The authors took advantage of constrained deformation in PDMS to develop a thermally actuated plastic microfluidic valve. The fluid flow is controlled through the deflection of a thin elastomeric film, actuated by a temperature-sensitive fluid located inside the valve. Heaters are manufactured by depositing a $100 \mathrm{~nm}$ thick gold film onto a cleaned plastic film by sputtering. Depending on the heater power used, the local channel temperature was 10 to $19{ }^{\circ} \mathrm{C}$ above the room temperature, enabling control of flow rates from 0.33 to $4.7 \mu \mathrm{L} / \mathrm{min}$ in a $110 \mu \mathrm{m}$ wide and $45 \mu \mathrm{m}$ deep microchannel.

Similarly, Gu et al. [76] used a PDMS based three-layer structure to control the opening/closing of a microchannel (Figure 9). This technique is also applicable to polymethylmethacrylate (PMMA). The valve-containing device can withstand about $700 \mathrm{kPa}$ without delamination, and the PDMS/PMMA bonding strength reaches a plateau when the temperature is higher than $70{ }^{\circ} \mathrm{C}$.

Finally, different temperature profiles may be required: either homogeneous as in PCR applications, or gradient-like for TGF or droplet actuation techniques. In both cases, it may be crucial to perform a temperature profile with the best achievable accuracy, although some applications do not require a sharp control. In order to meet such stringent requirements, different heating techniques and geometries of heaters have been investigated: the use of ionic liquids, in situ fabrication of wires and surface patterning of metal resistors using classical microelectronic techniques. These techniques are summarized in two larger categories: the generation of a homogeneous temperature profile and generation of a temperature gradient. 
The next two subsections are dedicated to spatial control of the temperature.

Figure 9. Process flow of bonding a thermoplastic substrate with a polydimethylsiloxane (PDMS) layer (a-d), followed by additional steps for valve fabrication (e-g). Reprinted with permission from [76]. Copyright 2011 American Chemical Society.

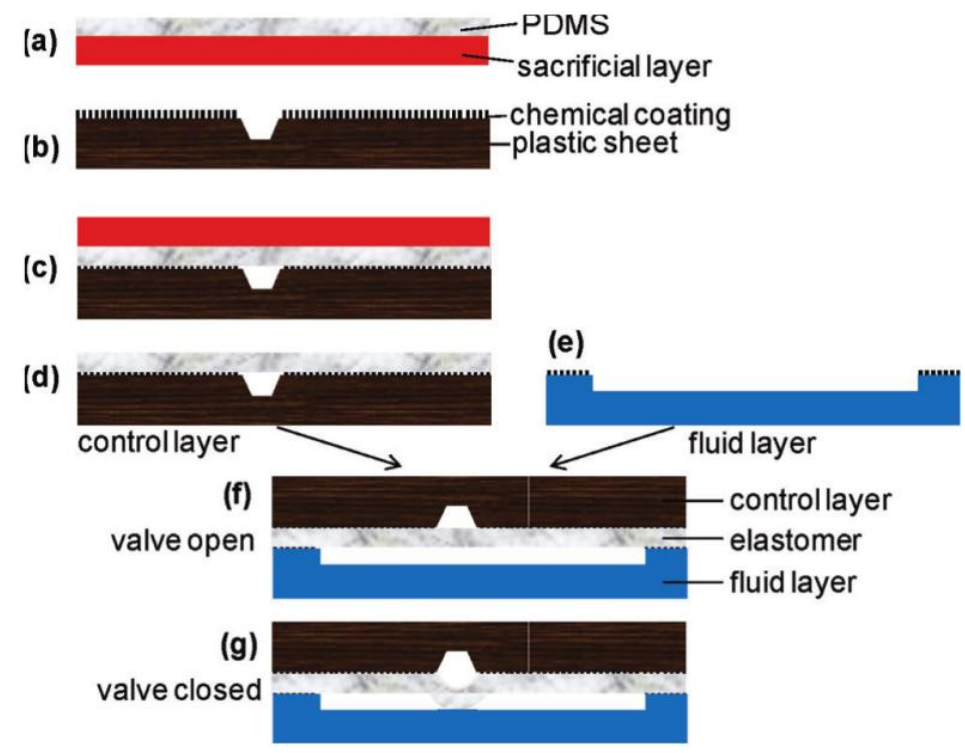

\subsection{Homogeneous Temperature}

To our knowledge, the only reported work using a conductive liquid is from De Mello et al. [41]. The authors present a microfluidic device incorporating working channels (sample) with a serpentine-like geometry and parallel channels (Figure 10) in which ionic liquids are Joule heated with an ac current (up to $3.75 \mathrm{kV}, \mathrm{f}=50 \mathrm{~Hz}$ and $\mathrm{P}=1 \mathrm{~W}$ ). Consequently, the internal temperature can be easily and directly controlled. Temperature measurements were performed using three thermocouples. The ionic liquids used in this experiment were [BMIM][PF6] and [BMIM][Tf2N]. Devices can be heated rapidly or slowly, depending on the applied voltage, and temperatures ranging from $50{ }^{\circ} \mathrm{C}$ to $90{ }^{\circ} \mathrm{C}$ can be set to within $\pm 0.2{ }^{\circ} \mathrm{C}$.

Figure 10. Sketch of the device composed of a working channel (depicted in black) together with parallel channels containing the conductive liquid (depicted in gray). Crosses stand for the position of thermocouples. Reprinted with permission from [41]. Copyright 2004, Royal Society of Chemistry.

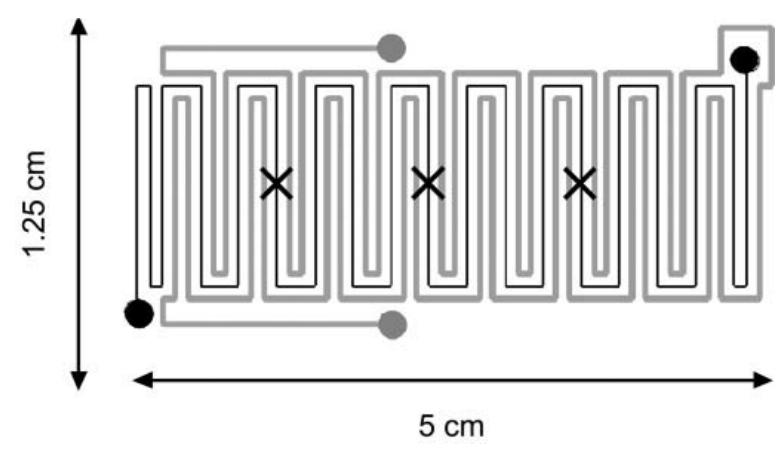


The serpentine-like geometry was also studied by Lao et al. [44] with integrated platinum heaters and sensors (Figure 11(a)), thermally isolated and digitally feedback controlled allowing a temperature control of $\pm 1{ }^{\circ} \mathrm{C}$ and rapid heating/cooling processes: (heating rate of $20{ }^{\circ} \mathrm{C} / \mathrm{s}$ and cooling rate of $10{ }^{\circ} \mathrm{C} / \mathrm{s}$, response time of approximately $5 \mathrm{~s}$ ). A feedback control, based on a gain scheduling control algorithm, is used to have an improved temperature response inside the chamber. The maximum power required to maintain a $20 \mu \mathrm{L}$ glycerol solution at $90{ }^{\circ} \mathrm{C}$ is $2.2 \mathrm{~W}$. Figure 11 (b) shows a good agreement between the chamber temperature and the set point over one cycle, demonstrating a good control of the overshoot.

Figure 11. (a) Integration of platinum heaters (serpentine-like geometry) together with the integration of sensors. (b) Temperature response of the reaction chamber for different fluids, showing the gain scheduling control algorithm efficiency. Reprinted from [44], Copyright 2000, with permission from Elsevier.

(a)
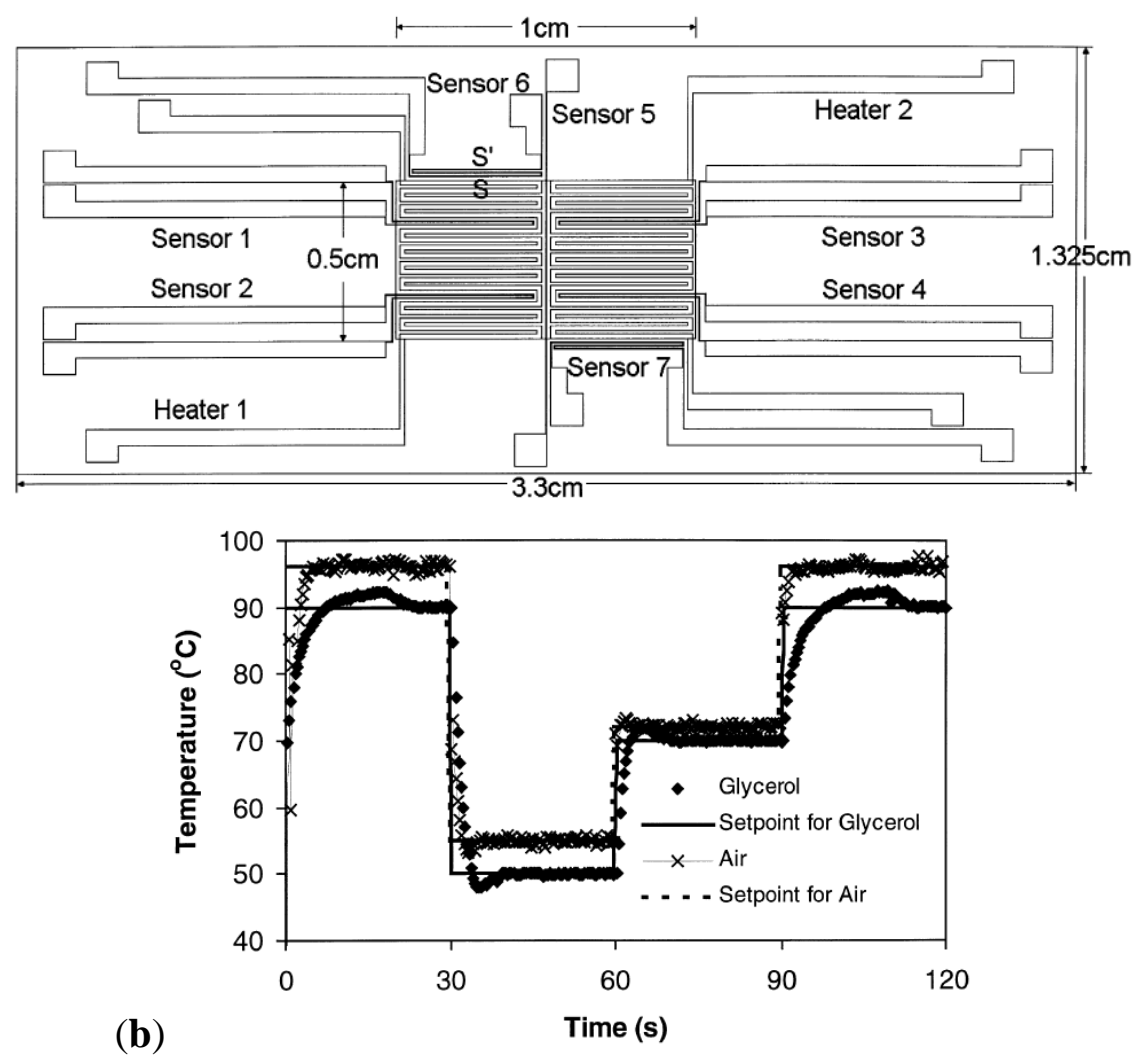

Based on the same heater geometry, Mavraki et al. [42] developed a simple microfluidic chip made of Pyralux ${ }^{\mathrm{TM}}$ with a double-sided Cu-clad polyimide (PI) $136 \mu \mathrm{m}$ thick substrate. PCR, with a fast DNA amplification rate, is performed. The DNA sample flows through the different thermal zones required to perform PCR (denaturation at $95{ }^{\circ} \mathrm{C}$, annealing at $60{ }^{\circ} \mathrm{C}$ and extension at $72{ }^{\circ} \mathrm{C}$, see Section 3.2) in a $150 \mu \mathrm{m}$ wide and $30 \mu \mathrm{m}$ deep microchannel, completing 25 thermal cycles and resulting in a $2^{25}$ multiplication factor of DNA. Each thermal zone is about $25 \mathrm{~mm} \times 10 \mathrm{~mm}$. This study shows a characterization of the microheaters used through the resistance versus temperature plot (Figure 8(a)).

Temperature control can be performed using platinum thin layers as heaters and as temperature sensors. Dinca et al. [8] presented a micro PCR reactor device using this type of heater. For the fastest 
experiment, 32 cycles were successfully carried out in less than $25 \mathrm{~min}$, with temperature ramps of $7.7{ }^{\circ} \mathrm{C} / \mathrm{s}$ for heating and $6.2{ }^{\circ} \mathrm{C} / \mathrm{s}$ for cooling. Lien et al. [9] presented an integrated microfluidic system capable of performing RT-PCR (Reverse Transcription of RNA to DNA previously to PCR: $70{ }^{\circ} \mathrm{C}$ during $10 \mathrm{~min}, 48{ }^{\circ} \mathrm{C}$ during $1 \mathrm{~h}$ and $95^{\circ} \mathrm{C}$ during $15 \mathrm{~min}$ ) processes for multiple simultaneous detections of four major types of aquaculture disease markers. Bloc platinum resistors are chosen as the material for the micro heaters and the temperature sensors, and gold ( $\mathrm{Au}$ ) metallization is used for the electrical connectors of both the micro temperature sensors and the array-type micro heaters (heating rate $20^{\circ} \mathrm{C} / \mathrm{s}$ and cooling rate $10^{\circ} \mathrm{C} / \mathrm{s}$ ).

Hsieh et al. [12] performed a rational approach by comparing the temperature response for different geometries of microheaters (Figure 12): two-blocks, two-blocks with additional side heaters, and an array with additional side heaters. Experiments show a temperature homogeneity improvement while increasing the number of heating sources for a given spatial region. An interesting matter raised by the authors is the level of accuracy while stating that the temperature is homogeneous on a whole cavity. As shown in Figure 12(c), it is obvious that a sensor placed at different locations (represented by gray lines) returns an average temperature smoothing the fluctuations along the sensor. Hence these experiments underline that stating a homogeneous temperature requires temperature mapping over the whole region of interest.

Figure 12. Infrared images of each microthermal cycler without heat sinks at the denaturing temperature. (a) 2-D temperature profile of the block-type microheaters. (b) 2-D temperature profile of the block-type microheaters with additional side heaters by applying an AC field. (c) 2-D temperature profile of the array-type microheaters with AC units. The dotted line shows the location of the PCR reaction chamber. The dimensions of each block in (a) are 2,900 $\mu \mathrm{m} \times 6,000 \mu \mathrm{m}$, which are divided into grids $(100 \mu \mathrm{m} \times 100 \mu \mathrm{m})$ with a spacing of $100 \mu \mathrm{m}$ in (c). Reprinted from [12], Copyright 2008, with permission from Elsevier.

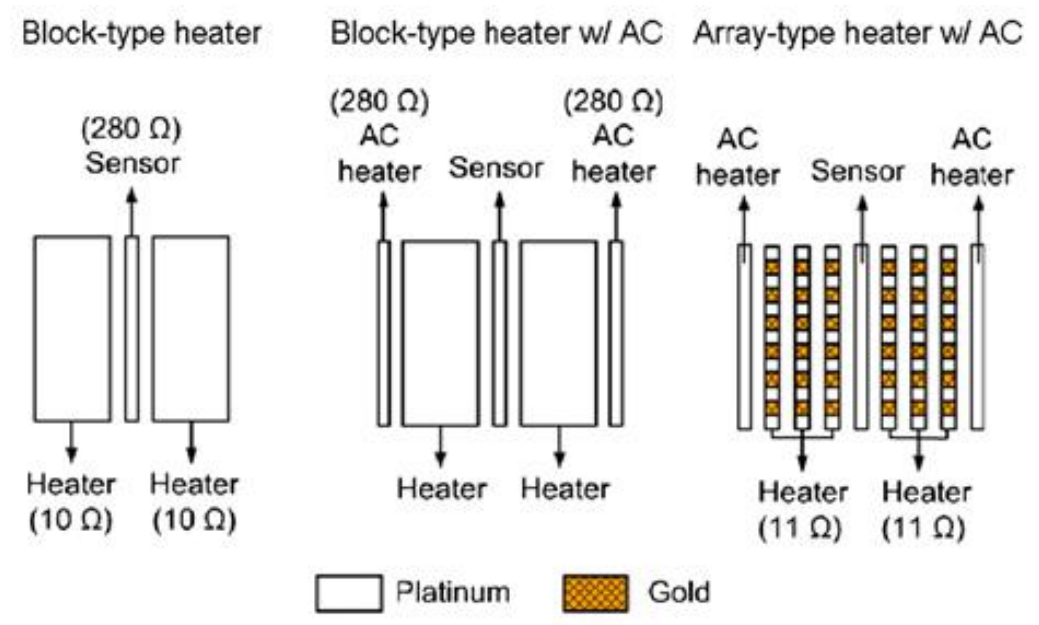

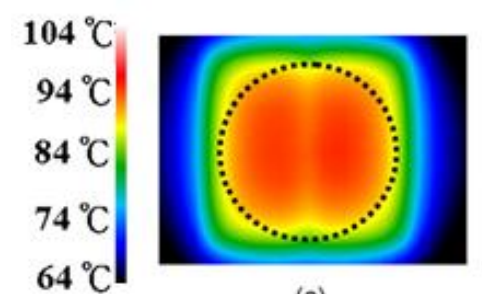

(a)

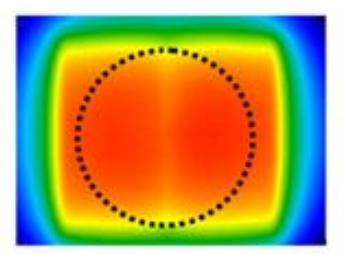

(b)

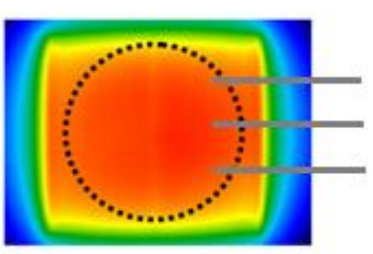

(c) 
The authors went deeper into their study by investigating other geometries such as serpentine-shape and self-compensated array-type heaters [13]. The aim of the study was to improve the temperature uniformity for PCR applications. Indeed, a homogeneous heater pattern cannot lead to a homogeneous temperature due to side effects, where thermal losses are higher than in the central zone of interest. The authors use electron-beam evaporation and standard lift-off processes to pattern thin-film heaters (90 nm Pt/15 nm Ti), a temperature sensor (90 nm Pt/15 nm Ti) and electrical leads (180 nm Au/20 nm Ti). Results show that a regular array gives a better homogeneity than two-blocks or serpentine, however this can be improved by a self-compensation: the heaters placed at the edges are smaller in order to counter-balance the side effects. The authors tested different self-compensations configurations. The self-compensated heaters happen to give the best uniformity on a selective region, with percentages of the uniform area of $90.3,99.9$ and $96.8 \%$ at 94,55 and $72{ }^{\circ} \mathrm{C}$ respectively, within thermal variation of $1{ }^{\circ} \mathrm{C}$.

This approach has been valued for PCR amplification by flowing reagents from one region, with a set temperature of $55^{\circ} \mathrm{C}$, to a warmer one (set temperature: $75{ }^{\circ} \mathrm{C}$ ). The microfluidic system contains three heating regions of different temperatures together with microfluidic channels. The temperature cycling is achieved by making a loop on the three regions. In 2009, Wang et al. [15] designed a microchip based on this principle. As shown in Figure 13, they designed three reaction open chambers (5 mm diameter) connected with microfluidic channels. Underneath, three array-type microheaters (Figure 12(c)) are patterned and delivered a homogeneous temperature profile. The liquid is displaced thanks to peristaltic valves [73] in approximately $2 \mathrm{~s}$. A cycle is performed in $110 \mathrm{~s}$. The main advantage of this method is the ease of temperature calibration and thus its precision.

Figure 13. IR images of the device showing the three different temperature zones $(5,72$ and $94{ }^{\circ} \mathrm{C}$ ). Reprinted from [15], Copyright 2004, with permission from Elsevier.

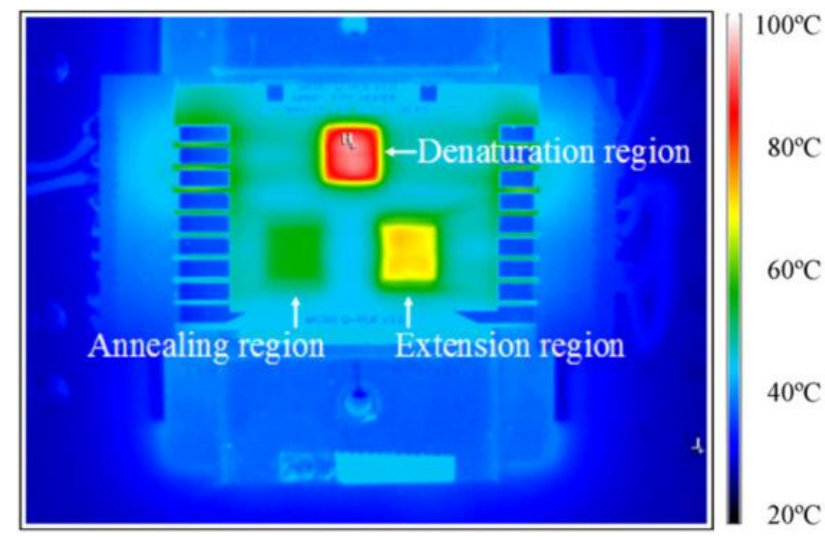

In the same spirit of shape optimization, Selva et al. [29,45] provided shape optimization of heating resistors in order to generate different temperature profiles. Shape optimization was carried out on the heating resistor shape, coupling two numerical tools: a genetic algorithm (NSGAII) [77,78], and a finite element study of the thermal response of the heaters. The resistors are made of chromium $15 \mathrm{~nm}$ thick. The typical heating power required is of the order of hundreds of $\mathrm{mW}$. A $600 \mu \mathrm{m} \times 600 \mu \mathrm{m}$ square region is heated at $49{ }^{\circ} \mathrm{C}$ with a transient regime of $2.2 \pm 0.1 \mathrm{~s}$ to reach an asymptotic state (90\% of the asymptotic value is reached in about $1 \mathrm{~s}$, which is much faster than a Peltier heater), see Figure 14(a,b) for which it is clear that side effects have to be compensated by thinner resistors at the 
edges. The cycling temperature was demonstrated as having good stability over time, provided a heat sink is placed below the cavity (Figure 14(c)).

By patterning the substrate with an optimized resistor, it is possible to generate a homogeneous temperature within a cavity with great accuracy and with short response times (standard deviation below $1{ }^{\circ} \mathrm{C}$ and asymptotic regime reached after $2.2 \mathrm{~s}$ ).

Figure 14. (a) Experimental temperature distribution resulting from the optimized resistor, providing a mean temperature in the cavity of $49{ }^{\circ} \mathrm{C}$; (b) Experimental temperature distribution in the non-optimized case (i.e., with constant-width elements), for a mean temperature into the cavity of $51{ }^{\circ} \mathrm{C}$. (c) Experimental mean temperature versus time for cycles with a $10 \mathrm{~s}$ period and an acquisition frequency of $25 \mathrm{~Hz}$. The transient state lasts approximately 2 s. Reprinted from [45], with kind permission from Springer Science+Business Media.
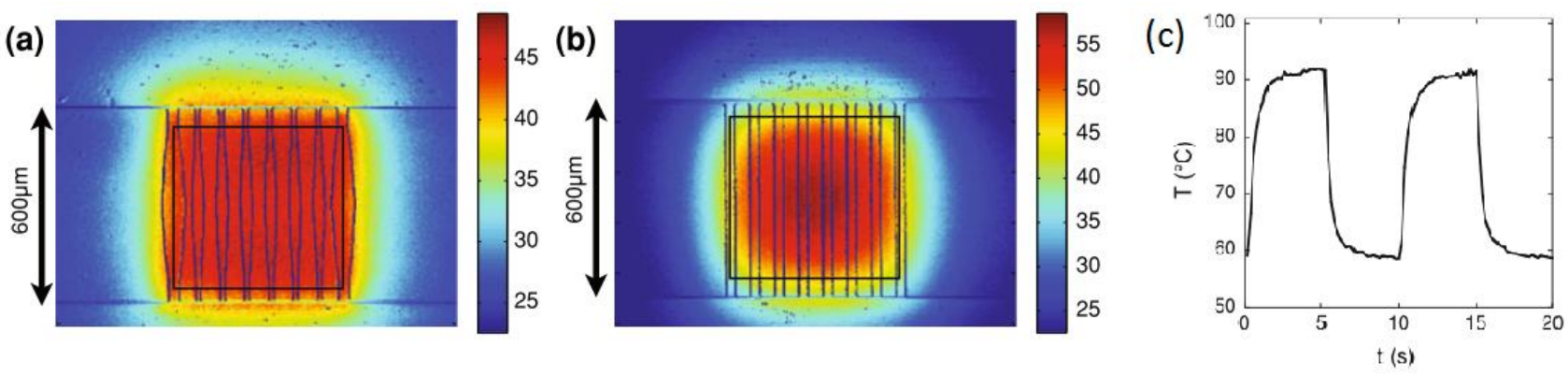

Figure 15. Temperature in the working channel as a function of the squared input voltage. The three insets are IR images illustrating the spatial distribution. Reprinted with permission from [67]. Copyright 2009, American Institute of Physics.

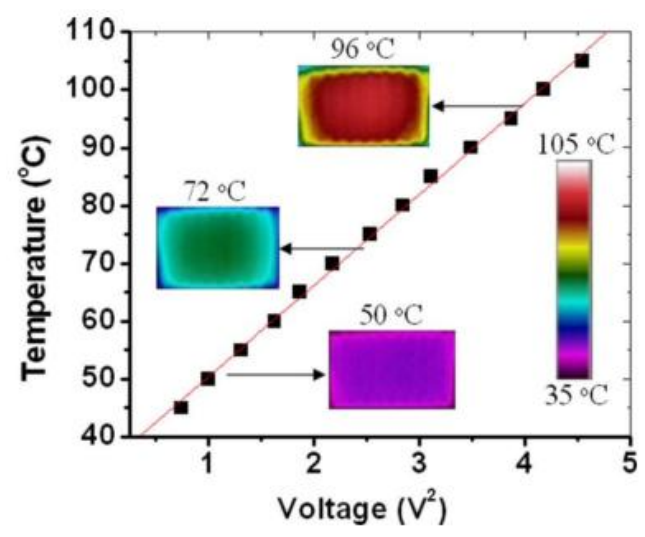

The last reported technique is the integration of metal wires. Wu et al. [67] designed a microheater and also thermal sensor directly by injecting silver paint (or other conductive materials) into a PDMS microchannel. In this study, they use SPI silver paste diluted by SPI thinner (ratio 1:3) followed by an ultrasonic bath treatment. The paste is injected in the channel and then heated to vaporize the solvent in three steps: $60{ }^{\circ} \mathrm{C}, 100{ }^{\circ} \mathrm{C}$ and $150{ }^{\circ} \mathrm{C}$. The calibration curve Resistance vs. Temperature is done with an IR camera and reveals a good spatial homogeneity in the middle of the serpentine. It also shows a good linearity in the $45-105{ }^{\circ} \mathrm{C}$ range. They achieved a heating rate of $20{ }^{\circ} \mathrm{C} / \mathrm{s}$ and a steady state error of about $\pm 0.5^{\circ} \mathrm{C}$. With an applied voltage varying from 0.9 to $2.2 \mathrm{~V}$, the authors obtained a 
temperature from 45 to $110{ }^{\circ} \mathrm{C}$ (Figure 15). Moreover, by measuring the resistance of a thinner wire, they could deduce its temperature. Finally, by designing a double serpentine (a large one for heating and a thin one for sensing), they created a microheater and a thermal sensor. Adding air-cooling channels, LabView voltage and air pressure controls (with a PID module), they finally designed a $25 \times 25 \mathrm{~mm}^{2}$ temperature controller that can be bonded under a micro-chip. One of the advantages of this technique is the low cost of the device.

\subsection{Temperature Gradient}

For given applications (e.g., droplet actuation, Soret effect, TGF, etc.) it is necessary to generate temperature gradients, either in a controlled way (controlled shape of the temperature profile) or not.

In the field of droplet-based microfluidics, a first application is focused on the displacement of a droplet in a capillary (1D geometry). Nguyen et al. [22] presented both theoretical and experimental results of thermocapillary effects of a liquid plug in a long capillary, subject to a transient temperature gradient generated by a resistive heater. The transient temperature gradient spreads in the capillary wall much slower than the droplet itself. Consequently, the plug moves out of the high-gradient region and decelerates. Jiao et al. [23] reported the reciprocating thermocapillary motion of a liquid plug located in a capillary and positioned between two heaters. The model shows the coupling effect between the surface tension driven movement of the plug and the heat transfer in the capillary wall. The temperature gradients, generated by the two heaters, cause a liquid motion. Finally, Shen et al. [14] investigated the physical mechanisms affecting migration of droplets due to thermocapillarity. A constant thermal gradient (up to $4.21{ }^{\circ} \mathrm{C} / \mathrm{mm}$ ) is generated by powering a metal heater stripe at one edge of the chamber, and cooling at the opposite edge by circulating coolant through a brass heat sink. The results of this study shed light on the critical role of mechanical or chemical hysteresis, and highlight the need to minimize power requirements in microfluidic devices.

Figure 16. (a) A schematic view of the microfluidic device (dimensions in $\mu \mathrm{m}$ ). (b) Variation of the delay distance $\mathrm{d}$ with temperature. Reprinted with permission from [18]. Copyright 2099, Institute of Physics.

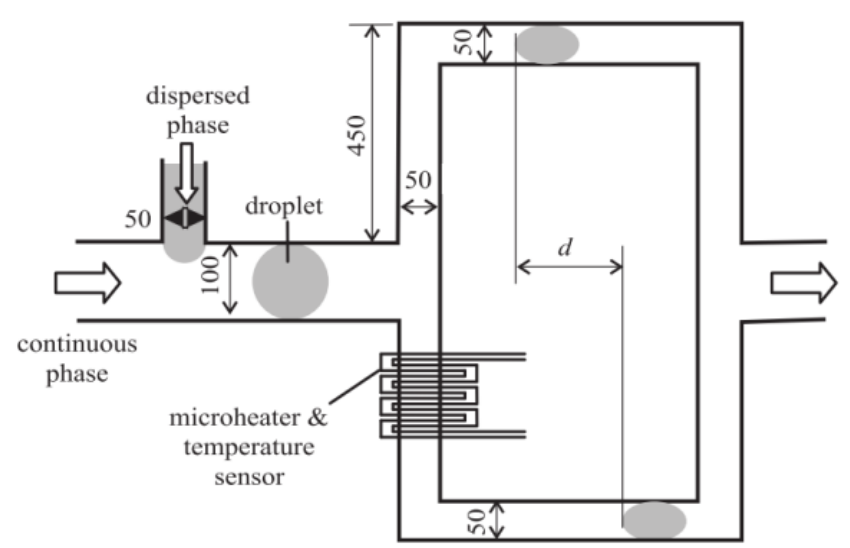

(a)

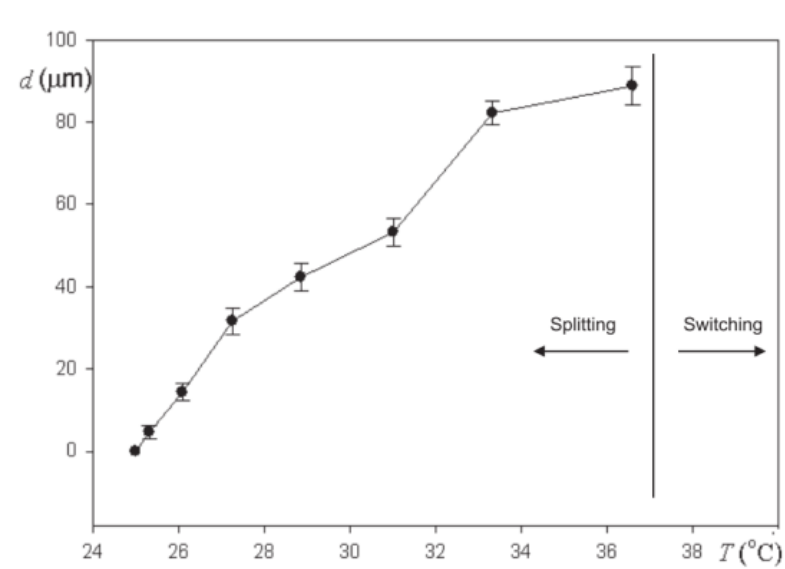

(b)

Another 1D droplet handling can be performed using the integration of a serpentine-like micro-heater which locally generates a temperature gradient together with a local decrease in the continuous phase 
viscosity. Considering such an integration in a 1D geometry, it is possible to control the breakup or switching of a droplet arriving in a T-junction as reported by Yap et al. [18,19]. The authors present a thermal control technique for microdroplets at a bifurcation, using an integrated microheater which induces simultaneously thermocapillarity and a reduction in fluidic resistance in one of the branches (Figure 16(a)). Droplet breakup and switching are demonstrated within a temperature range of 25-38 ${ }^{\circ} \mathrm{C}$ (Figure 16(b)), which enables dealing with biological samples.

Jiao et al. [20,21] presented a device with four integrated heaters providing temperature gradients for droplet-based microfluidic systems (Figure 17). The heaters are structured on a glass wafer of a $10 \mathrm{~mm} \times 10 \mathrm{~mm}$ square region and are made of thin-film titanium and platinum. The maximum heating power of each heater is equal to $0.5 \mathrm{~W}$.

Figure 17. Top and lateral views of the device showing four heaters placed along a square, generating temperature gradients, for which both shape and magnitude influence the heating power of each single heater. Reprinted with permission from [21]. Copyright 2008, Institute of Physics.

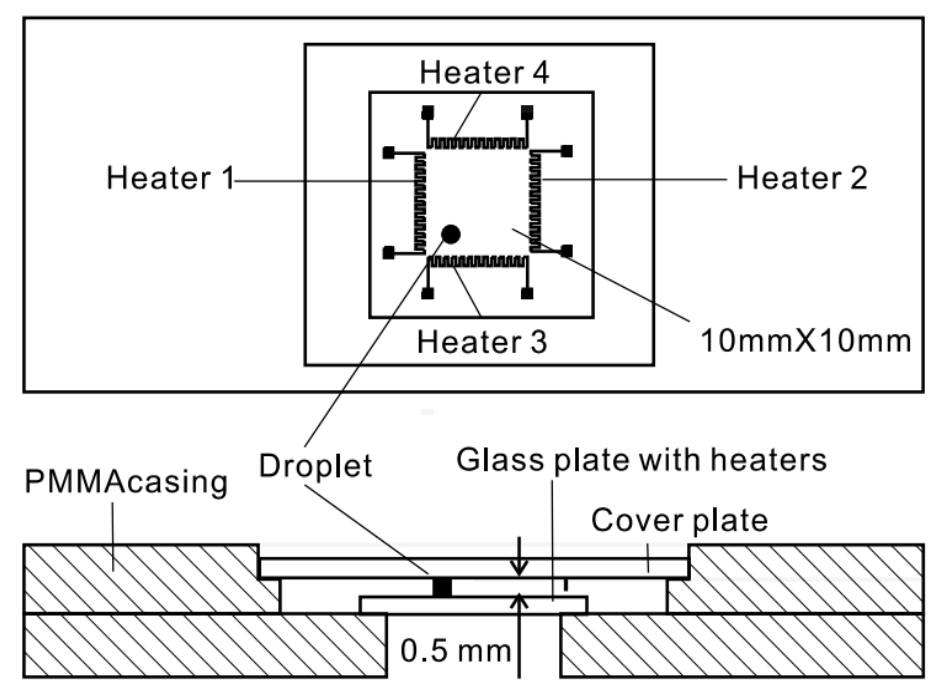

In such a configuration, it is possible to drive droplets by imposing a succession of different temperature gradients along the 2D substrate. The four microheaters actuated independently generate variable surface tension gradients. The droplet can be positioned anywhere in the channel depending on the strength of individual heaters (Figure 18).

At a more integrated level, Darhuber et al. [24-27] developed a system with thin Ti metallic microheaters (thickness $100 \mathrm{~nm}$, length $3 \mathrm{~mm}$ and $0.8 \mathrm{~mm}$ width, and $500 \mathrm{~nm} \mathrm{SiO}$ layer deposited for electrical heaters isolation ) coupled to a chemical patterned glass substrate and electronic actuation. The typical range of applied power for a single microheater is 5-200 W (maximum output voltage $10 \mathrm{~V}$; maximum output current $90 \mathrm{~mA}$ ). Based on thermocapillary actuation, they controlled, with a great accuracy, the formation, 2D displacement, coalescence and breakup of droplet on demand [27] (Figure 19). The initial volume of liquid is $3-16 \mu \mathrm{L}$.

Selva et al. [29] also reported shape optimization on resistors (chromium $15 \mathrm{~nm}$ thick, connected by gold wires $150 \mathrm{~nm}$ thick) to generate a linear temperature profile, as sketched in Figure 20. Applying a power ranging from 200 to $500 \mathrm{~mW}$, an intense temperature gradient (up to $11^{\circ} \mathrm{C} / \mathrm{mm}$ with a standard 
deviation of approximately $1 \%$ ) is generated (Figure 20). The transient regime of application of the gradient lasts about $250 \mathrm{~ms}$.

Figure 18. Succession of droplet positions by spatially varying the temperature gradient in time, (duration $80 \mathrm{~s}$ ). Reprinted with permission from [21]. Copyright 2008, Institute of Physics.

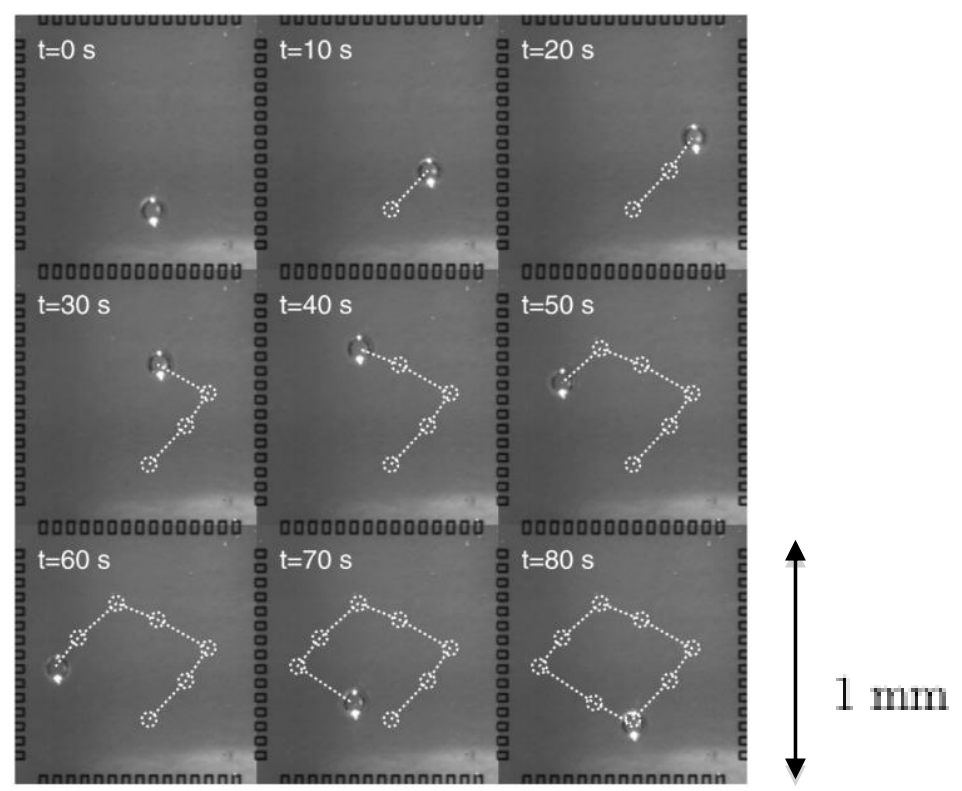

Figure 19. (a-e) Thermally induced splitting of a dodecane drop on a partially wetting stripe $(\mathrm{w}=1,000 \mu \mathrm{m})$. The voltage applied to the microheater $(155 \Omega)$ was $2.5 \mathrm{~V}$. The images were recorded at $\mathrm{t}=0,6.0,7.5,8.0$, and $8.5 \mathrm{~s}$. (f-i) Dodecane drop propelled through an intersection outlined by the dark gray pattern $(\mathrm{w}=1,000 \mu \mathrm{m}$, time lapse $104 \mathrm{~s})$. (j-l) Dodecane drop turning a $90^{\circ}$ corner (time lapse $164 \mathrm{~s}$ ). Reprinted with permission from [24]. Copyright 2003, American Institute of Physics.

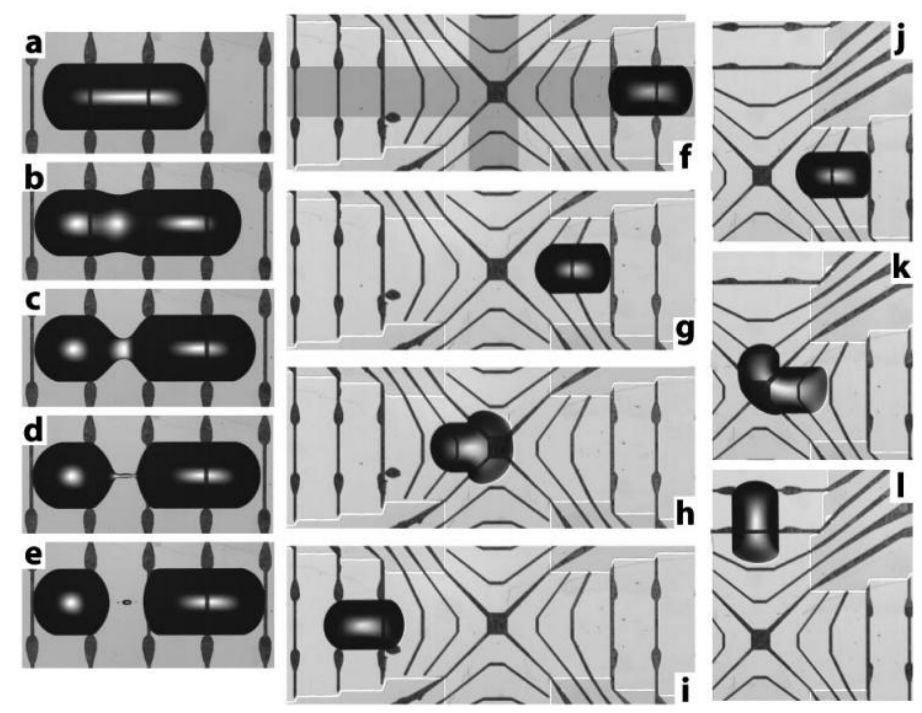


Figure 20. Top view of an output resistor geometry obtained performing shape optimization, made of chromium resistors (in gray) and gold connectors (in yellow). The experimental temperature profile along the cavity shows a linear dependence of the temperature with the $\mathrm{x}$-axis.

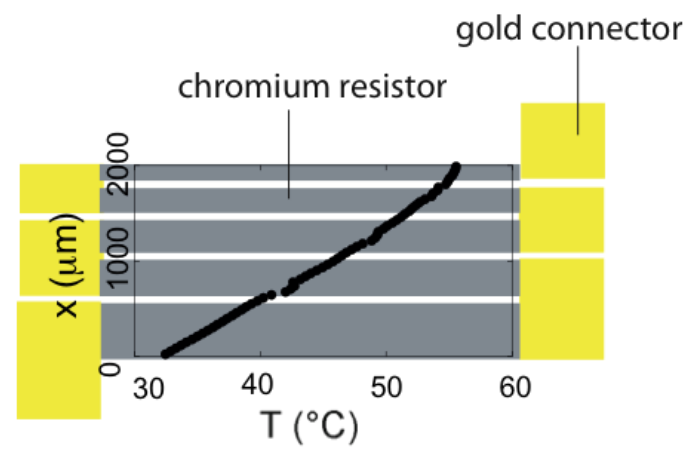

Using this resistor pattern, another phenomenon has been emphasized by Selva et al. [28]: thermomechanical effects due to PDMS dilation with increasing temperature. The authors present studies of pancake-like shaped bubbles in a Hele-Shaw cell, submitted to a temperature gradient [29]. Under such a confinement, there are mainly two competing mechanisms arising from the temperature gradient: thermocapillarity, and the thermal dilation of the PDMS cavity (Figure 21(a)). A theoretical model predicts the cavity dilation to be the dominant effect, which happens to be in excellent agreement with experimental results, inducing a bubble motion toward the cold region of the cavity. According to this study, Selva et al. [30] report a method for bubble and droplet displacement, switching (Figure 21(b)) and trapping based on a thermomechanical effect. This technique presents a high level of integration with low applied voltage $(\sim 10 \mathrm{~V})$ and low power consumption $(<0.4 \mathrm{~W})$. This work clearly highlights for the first time competing phenomena involved in microfluidics when changing the temperature.

Figure 21. (a) Sketch of the competition between thermocapillary and thermomechanical effects on bubble migration. Reprinted with permission from [28]; Copyright 2011, American Institute of Physics. (b) Images of a $300 \mu \mathrm{m}$ diameter bubbles inside a switching device: (left) without actuation, and (right) with a $4{ }^{\circ} \mathrm{C} / \mathrm{mm}$ temperature gradient. Reprinted with permission from [30]. Copyright 2010, Royal Society of Chemistry.
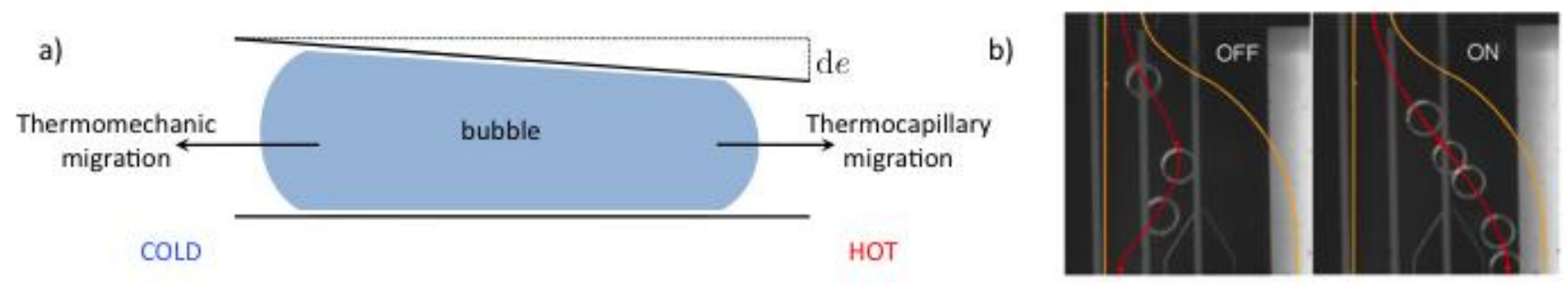

In order to generate a temperature gradient, copper blocks can also be integrated within a microsystem. Ross et al. [17] described such a system in which a temperature gradient is generated for TGF purposes (see Section 5). The device consists of two copper blocks set to different temperatures in order to generate a temperature gradient across a $2 \mathrm{~mm}$ gap microfluidic channel (Figure 22). The 
system is based on TGF, where temperature gradients of $25^{\circ} \mathrm{C} / \mathrm{mm}$ are produced by thermally anchoring a thin polycarbonate microchannel chip to alternately heated or cooled copper blocks. The technique is demonstrated for a large variety of analytes (fluorescent dyes, amino acids, DNA, proteins, etc.) and is capable of more than 10000-fold concentration of a dilute analyte.

Figure 22. Schematic drawing of the Temperature Gradient Focusing apparatus. Reprinted with permission from [46]. Copyright 2002 American Chemical Society.

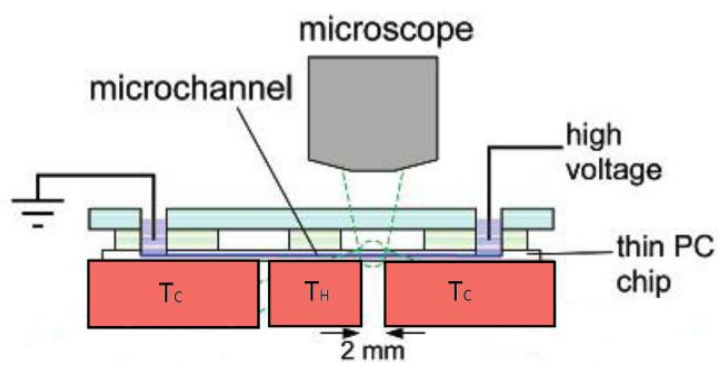

An interesting technique of embedded heaters is reported by Vigolo et al. [43]. The authors used a silver-filled epoxy (Epo-Tek_H20S, Epoxy Technology) that can be injected and solidified in a microfluidic chip, in parallel channels geometry. Applying an input current, both sides of a microchannel were heated by Joule effect. Depending on the geometry of the channels, either the control of a temperature gradient (Figure 23(a)) or the maintenance of a constant temperature (Figure 23(b)) can be achieved. This approach presents a fully embedded technique to control temperature, and permits working continuously from $25{ }^{\circ} \mathrm{C}$ to $75^{\circ} \mathrm{C}$ in a PDMS based microfluidic (accuracy $\pm 2-3{ }^{\circ} \mathrm{C}$ ). In the transient regime, the temperature increases within 10-20 s and reaches a stable value in less than one minute. A thermocouple in contact with a thin glass cover slip was used to measure the temperature. Authors could finally obtain the temperature of the strip by taking into account the thermal conductivity, thickness and cross-sectional area of the glass slide.

Figure 23. (a) Plot of the temperature along the channel surrounded by channels filled with an epoxy. (b) A schematic view of a device that is able to create a constant temperature. Reprinted with permission from [43]. Copyright 2010, Royal Society of Chemistry.

(a)

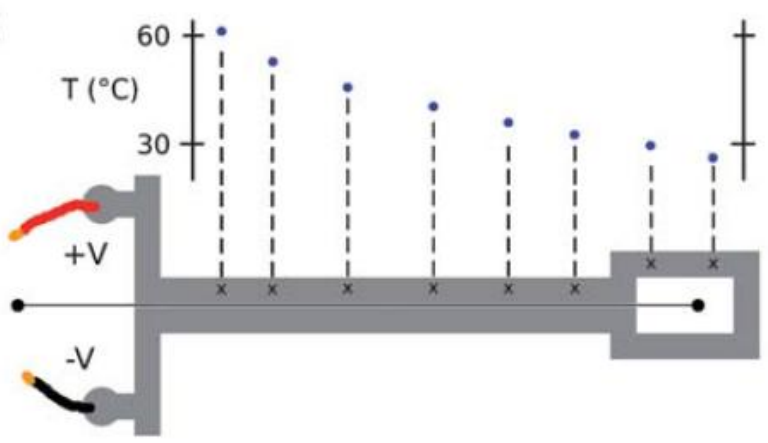

(b)

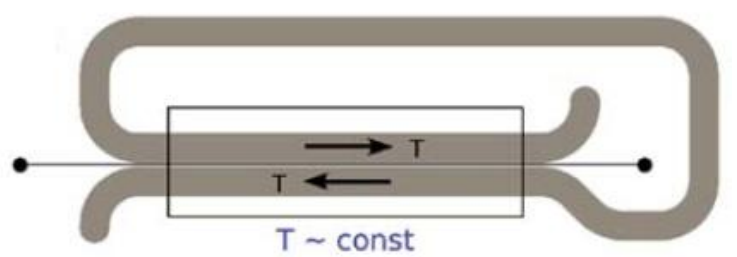

This technique can be combined with the pre-heated liquid technique as reported by Vigolo et al. [79] for thermophoresis studies (see Section 5). The authors describe a method for selective driving of particles towards either the hot or the cold side by adding specific electrolytes to their initial solution. The authors used a microfluidic device where temperature gradients were established by combining 
pre-heated liquid or epoxy resistors on either sides of the microchannel. Experiments bring into play the use of polystyrene beads of $477 \mathrm{~nm}$ in diameter in the presence of $100 \mathrm{mM} \mathrm{NaCl}$ with a flow rate of $0.01 \mu \mathrm{L} / \mathrm{min}$, and show the accumulation of particles on the cold side by fluorescence measurements.

Temperature gradient can also be used to generate natural convection for mixing purposes. Rapid and homogeneous mixing is difficult to achieve in microscale. Indeed, even if diffusion processes are favored in miniature fluidic systems, a pure diffusion-based mixing can be very inefficient, especially in solutions where macromolecules have a diffusion coefficient several orders of magnitude lower than that of most liquids. However, micromixing in chambers remains challenging even though many in-line micromixers have been developed and successfully demonstrated [32,34]. Kim et al. [33] presented an effective technique that enables micromixing in a microfluidic chamber without using a pump. By using natural convection in conjunction with alternating heating of two heaters (Figure 24), efficient micromixing is achieved. Heaters are made in a Ti/Pt alloy formed by a lift-off process, whose dimensions are typically $20 \mathrm{~nm} / 100 \mathrm{~nm}$ in thickness. Fluorescent microbeads of 8 - $\mu \mathrm{m}$ diameter were used as flow tracers to measure the flow speed at steady state. Standard deviation $\sigma=\left\langle(I-\langle I\rangle)^{2}\right\rangle^{1 / 2}$ was used to determine the degree of mixing in the chamber, where $\mathrm{I}$ is the normalized intensity of each pixel.

Figure 24. Natural convection-driven flows in a chamber. (a-d) Flow trajectories of fluorescent microparticles taken for $35 \mathrm{~s}$. Measured maximum temperatures, Tmax, in (a) to (d) are $52,51,46$, and $50{ }^{\circ} \mathrm{C}$, respectively. The yellow arrows indicate the gravity direction, and the white arrows depict the flow direction of the individual fluorescent particles of $8 \mu \mathrm{m}$ diameter. Scale bar, $1 \mathrm{~mm}$. Reprinted with permission from [33]. Copyright 2009 American Chemical Society.
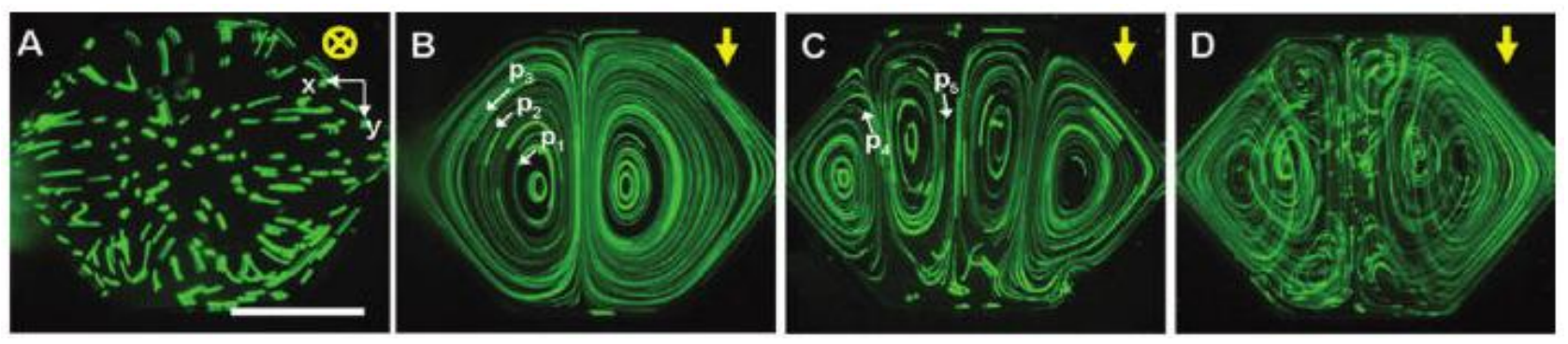

\section{Electromagnetic Radiation}

Up to now, we have seen that temperature control can be performed either in an external way or by integrating heating resistors. In both cases, heat diffuses from a source towards the liquid of interest. This section is devoted to techniques able to heat the liquid in the bulk, i.e., without using a thermal source.

\subsection{Microwaves}

Microwave dielectric heating is a fundamentally different approach because of its preferential heating capability and non-contact delivery energy. Induced and intrinsic dipole moments align themselves with a time-varying electric field (from 3 to $20 \mathrm{GHz}$ ). The energy associated is viscously 
dissipated as heat into the surrounding media with no interference from the substrate materials. Compared to conventional techniques, enhanced thermocycling rates and reduced reaction times can be achieved [54]. Heating can also be made spatially selective by confining the electromagnetic fields to specific regions thanks to integrating miniaturized microwave heating elements. The use of microwave heating has been demonstrated for a variety of applications including drug discovery [62], PCR [63] isolation of DNA and heating of biological cells [60].

Microwave power at several $\mathrm{GHz}$ is delivered to the channel by transmission line integrated in the microfluidics device. In most cases, a coplanar waveguide configuration is used. Shah et al. [59] carried out a study where a microchannel fabricated in PDMS [39] was aligned with a thin film microwave transmission line in a coplanar waveguide (CPW) configuration. A schematic of the device is shown in Figure 25(a). The CPW was comprised of a $140 \mu \mathrm{m}$ wide signal conductor separated by a $25 \mu \mathrm{m}$ gap on either side from $300 \mu \mathrm{m}$ wide ground conductors. The CPW conductors, $1.5 \mathrm{~cm}$ long, were formed by thermally evaporating $\mathrm{Cr} / \mathrm{Au}(10 \mathrm{~nm} / 500 \mathrm{~nm})$ on a $0.5 \mathrm{~mm}$ thick glass wafer and using a standard lift-off metallization process.

The device is characterized by the scattering $(\mathrm{S})$ parameter and the temperature. The S-parameters are the transmission and reflection coefficients. The fluid temperature is obtained by measuring the temperature dependent fluorescence intensity of a dilute fluorophore, Rhodamine $\mathrm{B}$, added to the fluid [46]. Figure 25(b) shows temperature vs. various microwave frequencies. Experimental data are compared to a theoretical model based on classical microwave absorption theory. They observed a $0.88^{\circ} \mathrm{C} \cdot \mathrm{mW}^{-1}$ temperature rise at $12 \mathrm{GHz}$ and $0.95^{\circ} \mathrm{C} \cdot \mathrm{mW}^{-1}$ temperature rise at $15 \mathrm{GHz}$. In agreement with the theory, they concluded that the temperature rise of the fluid is predominantly due to the absorbed microwave power. These results have been confirmed by recent works done on microwave dielectric heating of fluids [56].

Figure 25. (a) Lateral view of a coplanar waveguide transmission line (yellow) surrounding a PDMS microchannel (blue). (b) Measured temperature (squares) of an aqueous solution as a function of frequency compared with two theoretical models (solid and dashed lines). Reprinted with permission from [59]. Copyright 2007, Institute of Physics.

a)

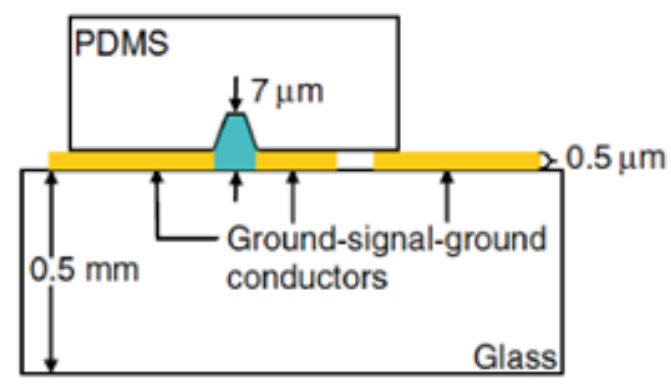

b)

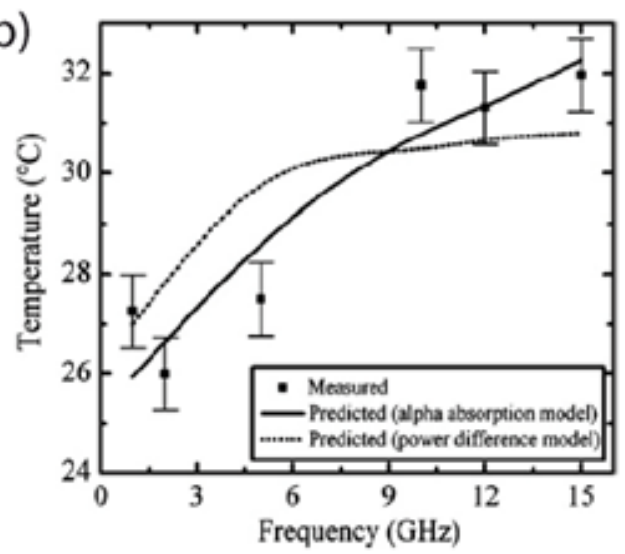

Kempitaya et al. [56] generated microwaves with millimetric copper strip transmission line (copper thickness $130 \mu \mathrm{m}$ ) put on top of a $1 \mathrm{~mm}$ thick polycarbonate substrate with a $2.3 \mathrm{~mm}$ diameter drilled well. Figure 26(a) shows such a device; the volume test chamber corresponds to $4.1 \mu \mathrm{L}$. They 
investigated the performance of microwave heating on a high temperature range, up to $70{ }^{\circ} \mathrm{C}$. The thermal response is recorded as a function of the applied microwave power for a prolonged period of time.

Figure 26. (a) Device schematic. (b) Temperature versus time for varying power levels. Reprinted with permission from [56]. Copyright 2009, American Institute of Physics.

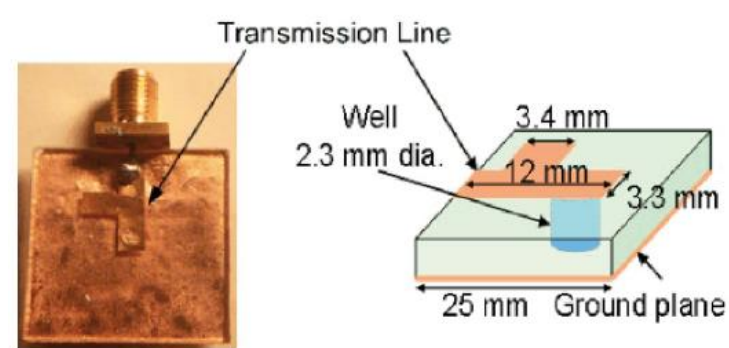

a)

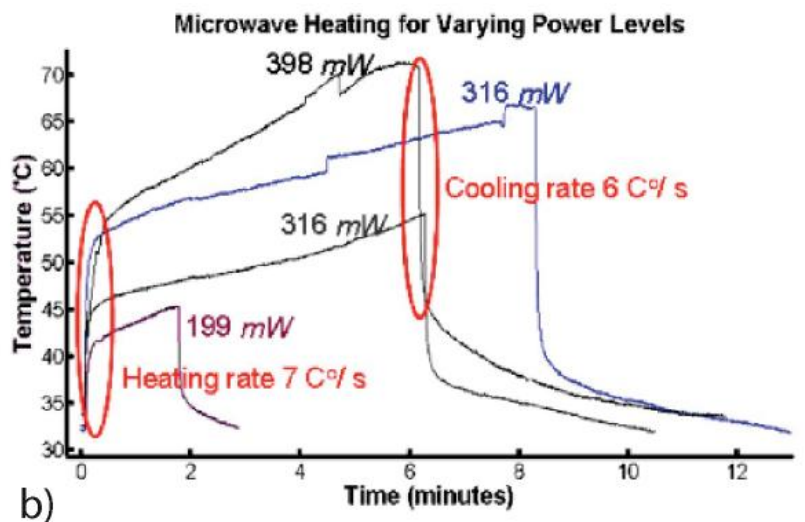

b)

They report two distinct heating/cooling behaviors (Figure 26(b)). During the first $30 \mathrm{~s}$ when the power is turned on (off), the heating (cooling) rate is very fast around $7{ }^{\circ} \mathrm{C} / \mathrm{s}\left(6^{\circ} \mathrm{C} / \mathrm{s}\right)$. The temperature is controlled by the heating/cooling of the microfluidic well (small thermal mass). However after this initial step, the heating/cooling rate decreases significantly, less than $0.1{ }^{\circ} \mathrm{C} / \mathrm{s}$. The substrate of the device, with a larger thermal mass, controls the temperature rise. A finite element transient heat transfer modeling matches the experimental results and gives the temperature profile along the well. Because of heat loss through the copper electrode the temperature of the liquid is only uniform over more than $50 \%$ of the chamber.

In the same spirit Shaw et al. [57] improved the integrated microwave heating device by adding air impingement cooling in order to do rapid PCR amplification. Indeed a previous study performed in 2004 showed the capability of doing PCR in a $15 \mathrm{~mL}$ reaction volume but the cooling was not integrated and required manual transfer into a thermally controlled block for each annealing step [58].

Using feedback controlled microwave heating coupled with air impingement cooling, the system showed minimal thermal overshoot or undershoot at any of the three set temperatures (see Figure 27). Once the microwave system had reached a set temperature the variation was less than $\pm 0.1{ }^{\circ} \mathrm{C}$. The ramp rates for heating and cooling exceeded $65{ }^{\circ} \mathrm{C} / \mathrm{s}$, allowing very fast transitions between temperatures. In conclusion, this study enabled 28 cycles to be performed in $42 \mathrm{~min}$. This is one order of magnitude faster than current commercial systems.

Microwaves heaters can be used to set the temperature for a PCR reaction. Orlling et al. [58] designed a millifluidic tool to prove this concept for samples volumes from 2.5 to $15 \mathrm{~mL}$. At this scale, Joule heating processes loose in efficiency due to a small surface to volume ratio. Consequently, the authors take advantage of the in situ heating of the device thanks to microwaves. Cycles in only $200 \mathrm{~s}$ are achievable for such a large amount of liquid thanks to extremely fast and smooth heating and cooling. Downscaling was performed by Fermer et al. [63] on volumes of about $100 \mu \mathrm{L}$, allowing a cycle duration of $144 \mathrm{~s}$ with a power of $100 \mathrm{~W}$. Recently, Shaw et al. [57] illustrated the viability of this technique at the microfluidic scale. A glass system is composed of a reaction chamber of $0.7 \mu \mathrm{L}$, 
which is supplied by a microchannel to bring the DNA sample and the PCR mix. They performed $90 \mathrm{~s}$ cycles with heating and cooling rates as fast as $65^{\circ} \mathrm{C} / \mathrm{s}$.

Figure 27. Thermal cycling profile based on selected temperatures of 92,58 and $71{ }^{\circ} \mathrm{C}$.

Reprinted with permission from [57]. Copyright 2010, Royal Society of Chemistry.

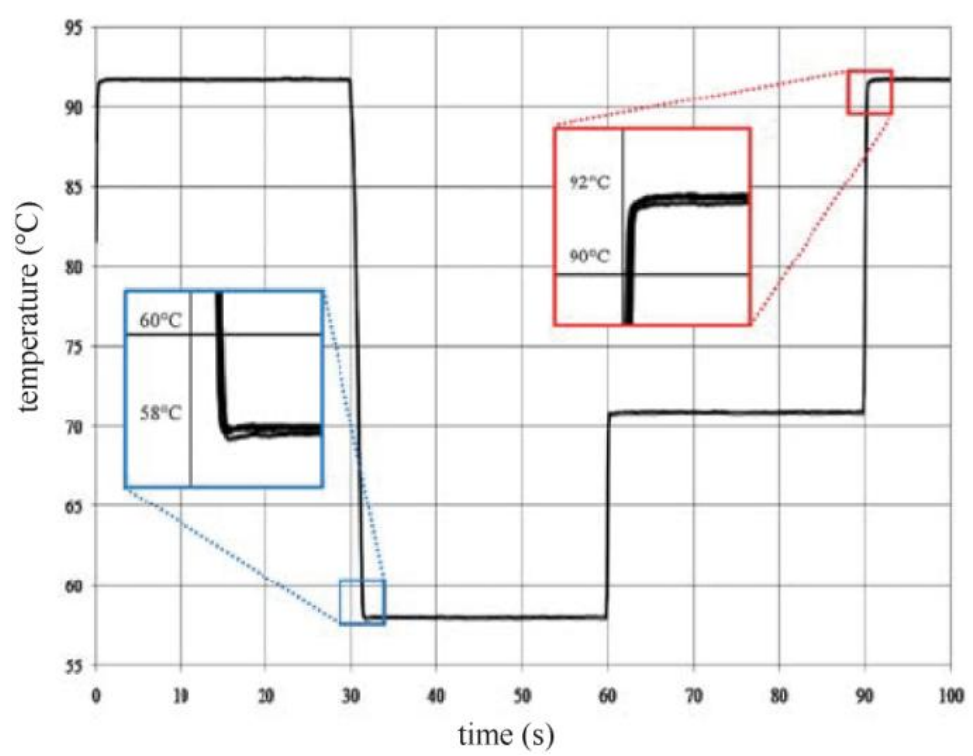

In the context of droplet-based microfluidic, Issadore et al. [64] reported the first experiment on microwave heating made on droplets. They used a microfluidic device that integrates a flow-focusing drop maker; drop splitters (Figure 28), and metal electrodes to locally deliver microwave power.

Figure 28. Picture of the device where the dark regions are the metal lines and the gray region in the middle is the channel. Reprinted with permission from [64]. Copyright 2009, Royal Society of Chemistry.

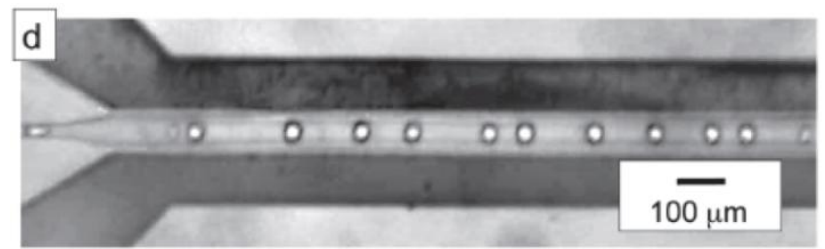

The devices are fabricated using PDMS on-glass drop-based microfluidics. The metal electrodes are directly integrated into the PDMS device using a low-melt solder fill technique [61]. The microwaves are generated with a voltage-controlled oscillator and amplified with a power amplifier. Copper wires approximately $2 \mathrm{~mm}$ in length connect the sub miniature assembly connector to the metal electrodes in the PDMS device. The electronics operate at $3.0 \mathrm{GHz}$. The temperature change of the drops is measured by observing the temperature-dependent fluorescence of cadmium selenide nanocrystals dispersed in the drops. With such a device they demonstrated characteristic heating times as short as $15 \mathrm{~ms}$ to steady-state temperature changes as large as $30{ }^{\circ} \mathrm{C}$ above the base temperature of the microfluidic device. In terms of applications the authors claim that setting the base temperature of the oil to $65{ }^{\circ} \mathrm{C}$, they could cycle the temperature from $65{ }^{\circ} \mathrm{C}$ to $95{ }^{\circ} \mathrm{C}$ as required for PCR. 
Up to now microwave heating has been used to generate homogeneous temperature and a fast heating rate. However spatial and temporal temperature gradients are also of primary interest in the field of enzymatic activity, thermodynamics, kinetics of molecular association, TGF and droplet handling to cite but a few. In their study, Shah et al. [55] used large interference effects to make a linear temperature gradient. Interference effects are produced by superposition of a sinusoidal and two exponential temperature distributions. Temperature extremes of $31{ }^{\circ} \mathrm{C}$ and $53{ }^{\circ} \mathrm{C}$ at the minimum and maximum of the sinusoid were established within $1 \mathrm{~s}$. The sinusoid also produced a quasi-linear temperature gradient along a $2 \mathrm{~mm}$ distance with a slope of $7.3^{\circ} \mathrm{C} \cdot \mathrm{mm}^{-1}$ (see Figure 29).

Figure 29. Measured temperature versus distance along the microchannel of an aqueous solution for two microwave excitation frequencies: (a) $19 \mathrm{GHz}$ and (b) $12 \mathrm{GHz}$. The solid red line is a theoretical temperature fit to the data points in squares. Reprinted with permission from [55]. Copyright 2010, Institute of Physics.

(a)

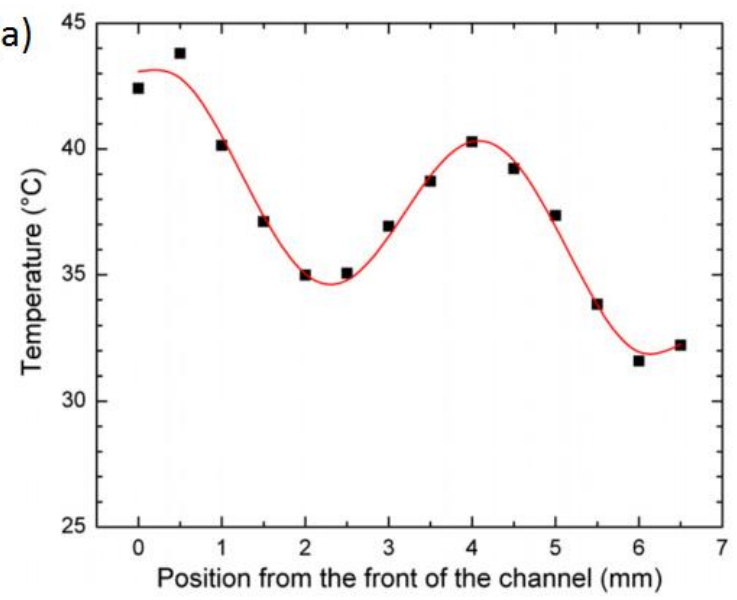

(b)

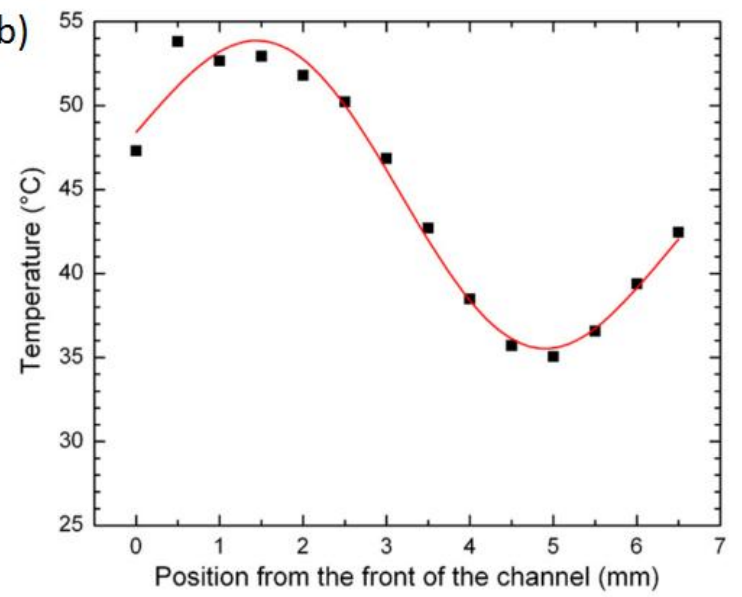

\subsection{Use of a Laser}

With the development of microelectronics, the laser diode became a cheap and reliable technology. The laser is now an easy and simple way to optically interact with a material. It is thus a rational approach in order to heat precisely low volumes. The spatial precision is easily around a micrometer and the volumes employed in microfluidics allow low consumption of products. Even if it is not an integrated method (for the moment, the laser remains out of the chip), it offers a cheap and spatially reconfigurable heat source that can be precisely addressed.

In their study, Robert de Saint Vincent et al. [68] used a laser as a heating source. The experiment consisted of focusing a continuous Argon-ion laser (wavelength in vacuum $\lambda_{0}=514.5 \mathrm{~nm}$ ) on a PDMS microchannel by using an inverted microscope. The aqueous phase is heated by adding $0.1 \mathrm{wt} \%$ of fluorescein (which gives an optical absorption of the solution $\alpha=1.18 \mathrm{~cm}^{-1}$, thus absorbing the laser radiation). The maximum thermal gradient that can be obtained at the edge of the beam is given by $\mathrm{P} / \mathrm{w}_{0}$ ( $\mathrm{P}$ is the power of the laser) and reaches (for $\mathrm{P}$ around $100 \mathrm{~mW}$ ) 10 to $20{ }^{\circ} \mathrm{C} / \mathrm{mm}$. The temperature gradient localized at the front of a droplet creates a surface tension gradient and induces droplet displacement in order to sort them. In Figure 30, one can see that for three different speeds (a-c), a $100 \%$ sorting of droplets is accomplished (water + dye drops in oil). When the laser is switched on, it locally heats the liquids (on an area about the beam waist) and thus creates a surface 
tension difference and induces a flow that moves the drop due to Marangoni stress. Baroud et al. [80] used the same concept with a flow focusing geometry to control the production of droplets.

Kim et al. [69] also used a laser in order to heat nanoliter drops and to perform real-time PCR. Their laser is an infrared diode at a wavelength of $1.46 \mu \mathrm{m}$, which corresponds to the first overtone of the O-H stretch vibration of liquid water. The beam size is about $200 \mu \mathrm{m}$ with a $10 \times$ objective in order to heat $300 \mu \mathrm{m}$ diameter droplets. To perform the PCR, they use two powers: 25 and $50 \mathrm{~mW}$ to achieve 60 and $95{ }^{\circ} \mathrm{C}$. The accuracy of the steady state is less than $1{ }^{\circ} \mathrm{C}$. They perform a complete amplification in $20 \mathrm{~s}$. This method can be coupled to a powerful laser with a microlens array in order to realize real-time PCR over hundreds of droplets.

Ohta et al. [70] used this tool to control the displacement of a gas bubble in silicon oil via thermocapillary forces. Contrary to other works, the temperature gradient is generated by the laser absorption in the silicon substrate and not in the liquid. In order to create optically actuated thermocapillary forces, they used an absorbing substrate consisting of a 0.85 -mm-thick glass slide coated with a 100 -nm-thick layer of indium tin oxide, followed by a $1-\mu \mathrm{m}$-thick layer of hydrogenated amorphous silicon $(a-\mathrm{Si}: \mathrm{H})$, which absorbs light in the visible and UV wavelengths. The laser power used is $10 \mathrm{~mW}$, and its wavelength is $635 \mathrm{~nm}$. The magnification is 20 and the beam obtained is a $6 \mu \mathrm{m}$-diameter spot on the surface of the absorbing substrate. They managed gradients in the surrounding oil up to $4{ }^{\circ} \mathrm{C} / \mathrm{mm}$. As one can see in Figure 31, the bubble is first trapped at the hottest point (because the bubble moves toward the warmest region) and then the laser spot is moved. This technique shows a good control of the displacement and moreover, it allows the use of fluids without any optical restrictions.

Figure 30. Superposition of successive frames illustrating drop switching by local thermocapillary actuation. The arrow indicates the laser location observed by the fluorescence of the water-dye solution. (a) $\mathrm{Qo} / \mathrm{Qw}=5 / 0.05, \mathrm{U}=2.6 \mathrm{~mm} / \mathrm{s}, \mathrm{R}=60 \mu \mathrm{m}$, $\mathrm{P}=58 \mathrm{~mW}$, frame rate 50 frames/s. (b) $\mathrm{Qo} / \mathrm{Qw}=10 / 0.1, \mathrm{U}=6.1 \mathrm{~mm} / \mathrm{s}, \mathrm{R}=54 \mu \mathrm{m}$. $\mathrm{P}=111 \mathrm{~mW}$, frame rate 100 frames $/ \mathrm{s}$. (c) $\mathrm{Qo} / \mathrm{Qw}=15 / 0.2, \mathrm{U}=13.0 \mathrm{~mm} / \mathrm{s}, \mathrm{R}=55 \mu \mathrm{m}$. $\mathrm{P}=159 \mathrm{~mW}$, frame rate 100 frames/s. Flow rates are expressed in $\mu 1 / \mathrm{min}$. Reprinted with permission from [68]. Copyright 2008, American Institute of Physics.

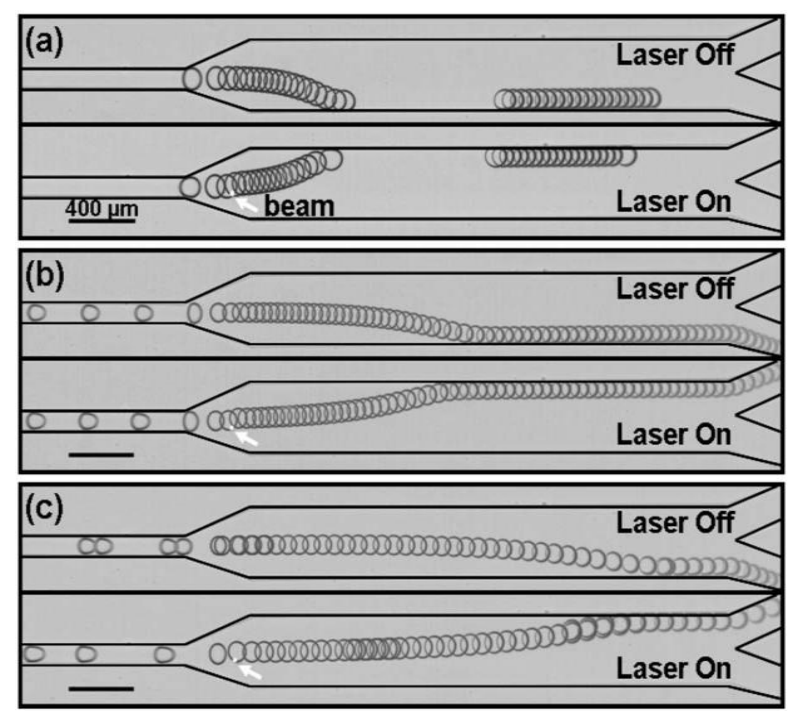


Another application stemming from the use of a laser was reported by Weinert et al. [81]. The authors were able to pump selected parts of a fluid film along the path of a moving warm spot which was generated by an infrared laser focus. The maximal temperature increase in the local spot is about $10{ }^{\circ} \mathrm{C}$ in water, corresponding to pump speeds of $150 \mu \mathrm{m} / \mathrm{s}$. The enhanced temperature in the spot leads to a reduced viscosity: experiments confirm that the fluid motion results from the dynamic thermal expansion in a gradient of viscosity. Consequently, the fluid moves opposite to the spot direction. Using this technique, the authors were able to pump nanoparticles over millimeters through a gel, and mixing was demonstrated for fluids sandwiched between untreated and unstructured, disposable microscope cover slips.

Hettiarachchi et al. [82] presented an optical microfluidic platform for performing real-time polymerase chain reactions of breast cancer cell DNA within droplet-in-oil microreactors. Droplet manipulation and rapid thermal cycling were achieved by using a low power $(20-40 \mathrm{~mW})$ infrared laser (lambda_0 = 1,460 nm). Droplet temperatures were calibrated based on fluorescence measurements of SYBR Green as well as amplification efficiency. The typical droplet diameter is about $200 \mu \mathrm{m}$ and fluorescence images are acquired at a $400 \mathrm{~ms}$ acquisition time. The illumination source was blocked with an automated shutter to avoid photobleaching. The authors also addressed the problematic of achieving spatial homogeneity. Indeed, the authors initially ran into problems with temperature non-uniformity across the droplets that impacts amplification efficiency. As a result, a 4-f lens configuration was used to defocus the laser beam to achieve a more uniform temperature distribution.

Figure 31. Optically actuated thermocapillary movement of an air bubble in silicone oil. A $114-\mu$ m-diameter $(1.0 \mathrm{~nL})$ bubble is trapped in the thermal trap created by a laser focused on the absorbing substrate (a). The oil/air meniscus can be seen at the top left. The bubble follows the position of the laser spot, as it is scanned in the positive y direction (b) and then in the positive $x$ direction (c). The bubble is then moved in the negative $y$ and negative $x$ directions (d). A dashed circle indicates the initial bubble position, and a dashed line indicates the approximate trajectory of the bubble. Reprinted with permission from [70]. Copyright 2007, American Institute of Physics.

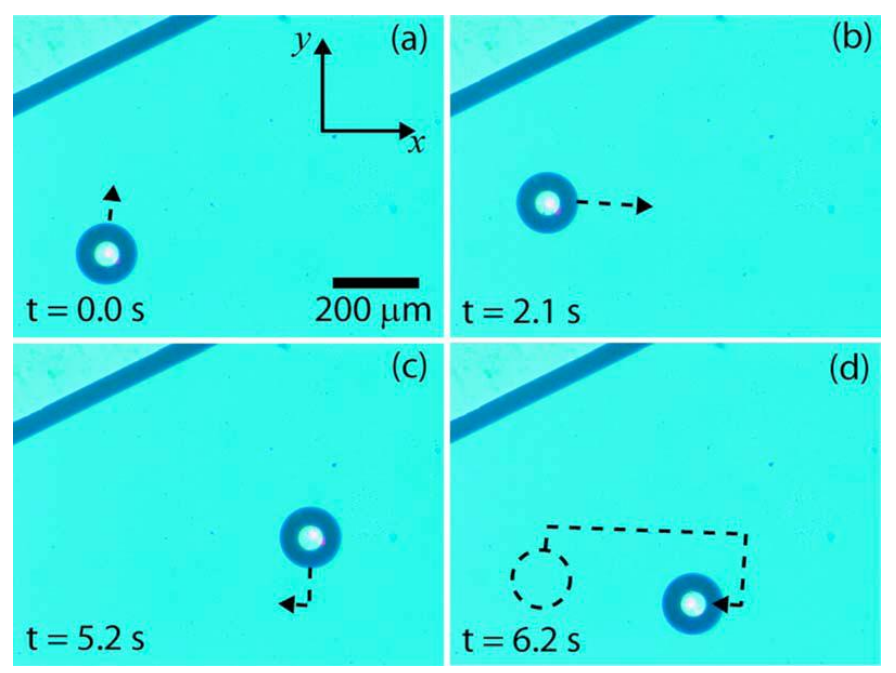




\section{Applications}

This section introduces a series of applications to illustrate the efficiency of reported heating techniques. We have tried as much as is possible to cover a range of different fields from the chemical, physical and biological sciences, without being exhaustive in any one domain; our purpose is rather to explore the diversity of microfluidic applications exploiting thermal control. The strategies and approaches vary from one application to another; while spatial temperature homogeneity is imperative for many biological applications; a rough temperature gradient is sufficient for most applications in digital microfluidics and a net temperature gradient is required for Temperature Gradient Focusing (TGF) and thermophoresis.

For biological applications, two main requirements have been reported: (1) maintaining the temperature at $37{ }^{\circ} \mathrm{C}$ to keep cells alive [83], the effect of temperature on the response of cells to stimuli can be obviously studied, however a homogeneous temperature is required; or (2) controlled temperature cycling for Polymerase Chain Reactions. In both cases, spatial homogeneity of the temperature profile is desirable. Polymerase Chain Reaction (PCR) is widely used in molecular biology to amplify target DNA in vitro [1]. A PCR cycle usually consists of three discrete steps, each step being highly temperature sensitive. In the case of DNA, a denaturation step performed at 94-96 ${ }^{\circ} \mathrm{C}$ causes DNA melting yielding single-stranded DNA molecules. Subsequently, an annealing step is carried out at $50-65^{\circ} \mathrm{C}$ : during which the polymerase attaches to a single-strand and begins DNA formation. Finally, the elongation or extension step is performed at $72{ }^{\circ} \mathrm{C}$ and DNA polymerase synthesizes a new DNA strand complementary to the single-strand template. Since PCR steps are short, the duration of a total amplification process with a commercially available thermal cycler is limited by the heating/cooling rates. Consequently, a thermal technique is needed to rapidly increase the heating/cooling cycling speed. The number of groups working on PCR worldwide is significant given the important social impact of this technique for example in the field of medical diagnostics. This paper does not aim to review all the systems that have been developed but rather to present specific approaches that have been reported [30,79]. Two key strategies have been adopted to facilitate thermal cycling: the PCR process is either performed in a single chamber where temperature is modulated over time (single heater device), or three independent heating zones in the microsystem (multiple heater devices) are integrated. In the latter case, solutions carrying the reagents either flow through the three regions with residence times matching the corresponding PCR step (multiple heater device), or are incubated in a preset temperature chamber for the required time.

Another approach is the generation of temperature gradients to drive droplets. Indeed, the use of droplet-based microfluidics [31] is largely presented as a viable alternative to handle small volumes (typically a few hundreds of a nanoliter). Micro droplets can be considered as perfect discrete biological reactors as they minimize cross-contamination, provided the partition coefficients prevent diffusion through the droplet interface [84]. Droplets also exhibit mixing by rolling recirculation that homogenizes the content, and can thus be considered as bona fide microreactors. The growing demand for handling picoliter to nanoliter volumes of biological samples has driven the development of droplet techniques where a variety of processes, including mixing, splitting and heating are efficiently controlled: these approaches have been designated as digital microfluidics. Such a prospective, demands tight control of fundamental operations such as droplet merging, fission, transport, exchanges, 
redirection, and storage. This has rekindled interest in the Marangoni surface effect, which refers to tangential stresses induced along an interface by a surface tension gradient [85]. The primary mechanism at play here is thermocapillarity, and bubbles or droplets are driven by the flow resulting from a gradient along their surface. Methods to induce temperature gradients in droplet systems are described and relatively coarse temperature gradients may be sufficient to drive droplets in these microsystems. This approach is an alternative to the Electrowetting On Dielectric (EWOD) technique [86] which refers to Maxwell stress applied to the triple line contact. The local electric field changes the local curvature and induces droplet motion. Although in the thermocapillary and EWOD, droplet motion is achieved with potentials of about $10 \mathrm{~V}$, EWOD requires optimizing dielectric thicknesses and properties [87].

Conversely, the following applications, which focus on chemical applications, demand a net temperature gradient, i.e., a constant temperature gradient. The detection of chemical species at small concentrations (nanomolar or lower) in small volumes (typically a few microliters or less) is a core functionality of miniaturized bioanalytical devices. Temperature Gradient Focusing (TGF), on the

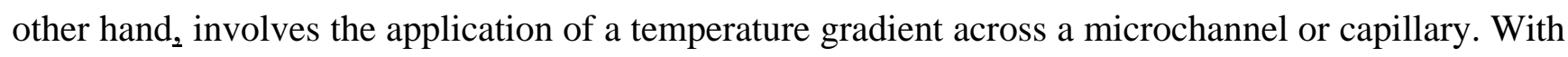
an appropriate buffer, the temperature differential creates a gradient in both the electric field and the electrophoretic velocity. Ionic species can thus either be concentrated by balancing the electrophoretic velocity against the bulk flow, or separated according to their individual electrophoretic mobilities. In other words, tuning the temperature allows modulation of the electrophoretic mobility at will. Another application centered on the migration of small particles, is thermophoresis. Thermophoresis (Soret effect) is the phenomenon wherein small particles suspended in a fluid with a temperature gradient, experience a force in the direction opposite to that gradient $[88,89]$. This effect can be used to drive particles in microfluidic devices and presents several advantages in terms of selectivity, due to its sensitivity to particle interfacial properties.

Finally, thermal control has found application in a broad gamut of diverse fields such as the development of efficient and rapid mixing techniques (for example solutions with low Reynolds number), or the screening of solubility diagrams to study protein crystallization.

\section{Conclusions}

Applications concerned with the control of temperature are numerous and focus on physical, chemical and biotechnological issues. This article shows the great variety of technologies that have been developed to achieve integrated temperature control. All these techniques present different advantages or drawbacks in terms of easiness of integration, cost, area of control, accuracy of the control, etc. that are summarized in the following table. This table underlines the fact that despite there being no paradigm to implement microheaters, a huge improvement has been performed in terms of level of integration, cost and response time showing very good ability to integrate the focused application. However, an important feature has been emphasized by several authors [12,13,28-30,45,82] concerning temperature measurement. Indeed, it has been shown that to generate a homogeneous temperature profile within a cavity or within a droplet, whole mapping of the temperature has to be performed since a sensor simply outputs a mean value. Even if a small standard error is not crippling for PCR applications, it can be so when using cells where biological activity is extremely sensitive to 
slight temperature variations. The development of new heating techniques will require focus on generating such accuracy.

As stated in the introduction, several strategies have been adopted to control the temperature within a microsystem, and most of the focused applications have been successfully achieved. It is thus difficult to isolate a technique that could be a paradigm. However, for some applications it is possible to extract a technique - that seems to be more accurate and show a high level of integration. In the case of droplet handling, Darhuber et al. [24-27], using chemical patterning and thin metal wires, were able to dispense, merge, break and drive droplets on a substrate. In this case a temperature pulse generates a temperature gradient sufficient to perform the expected functions; i.e., an accurate temperature profile is not necessary. For biological applications requiring a homogeneous temperature at the cavity level, Joule heating connected with shape optimization to avoid side effects has been shown to be very efficient with a high level of integration $[12,13,45]$. At the droplet level, IR heating is also very efficient, provided the beam is defocused to guarantee spatial temperature homogeneity in the droplet [82]. Finally, for screening solubility diagrams for which a temperature gradient is required, apart from the use of Peltier, techniques have been developed at a high level of integration such as shape optimization [29], such an approach could be used in the future. All these technologies are mature; nevertheless one may envisage in the future a technology embedding a heating source such as a conductive PDMS. Cong and Pan [90] succeeded in performing a thermal conductivity of about $80 \mathrm{~W} \cdot \mathrm{m}^{-1} \cdot \mathrm{K}^{-1}$ by adding $21 \%$ of silver powder. Such technology would avoid extra steps in the microfabrication process but would require optimization of thicknesses in order to proceed to optical measurements.

\section{Acknowledgments}

The authors wish to thank all the authors and publishers who agreed to reproduce the figures. We thank G. Fox for careful reading and suggestions. The authors are grateful to DGA, IPGG, CNRS, ESPCI and CEA for funding.

\section{References}

1. Bartlett, J.M.S.; Stirling, D. A short history of the polymerase chain reaction. Meth. Mol. Biol. 2003, 226, 3-6.

2. Maltezos, G.; Gomez, A.; Zhong, J.; Gomez, F.A.; Scherer, A. Microfluidic polymerase chain reaction. Appl. Phys. Lett. 2008, 93, 243901:1-243901:3.

3. Maltezos, G.; Johnston, M.; Taganov, K.; Srichantaratsamee, C.; Gorman, J. Exploring the limits of ultrafast polymerase chain reaction using liquid for thermal heat exchange: A proof of principle. Appl. Phys. Lett. 2010, 97, 264101:1-264101:3.

4. Khandurina, J.; McKnight, T.E.; Jacobson, S.C.; Waters, L.C.; Foote, R.S.; Ramsey, J.M. Integrated system for rapid PCR-based DNA analysis in microfluidic devices. Anal. Chem. 2000, 72, 2995-3000.

5. Yang, J.; Liu, Y.; Rauch, C.B.; Stevens, R.L.; Liu, R.H.; Lenigk, R.; Grodzinski, P. High sensitivity PCR assay in plastic micro reactors. Lab Chip 2002, 2, 179-187. 
6. Hua, Z.; Rouse, J.L.; Eckhardt, A.E.; Srinivasan, V.; Pamula, V.K.; Schell, W.A.; Benton, J.L.; Mitchell, T.G.; Pollack, M.G. Multiplexed real-time polymerase chain reaction on a digital microfluidic platform. Anal. Chem. 2010, 82, 2310-2316.

7. Mahjoob, S.; Vafai, K.; Beer, N.R. Rapid microfluidic thermal cycler for polymerase chain reaction nucleid acid amplification. Int. J. Heat Mass Transfer 2008, 51, 2109-2122.

8. Dinca, M.P.; Gheorghe, M.; Aherne, M.; Galvin, P. Fast and accurate temperature control of a PCR microsystem with a disposable reactor. J. Micromech. Microeng. 2009, 19, 065009:1-065009:15.

9. Lien, K.Y.; Lee, S.-H.; Tsai, T.-J.; Chen, T.-Y.; Lee, G.-B. A microfluidic-based system using reverse transcription polymerase chain reactions for rapid detection of aquaculture diseases. Microfluid. Nanofluid. 2009, 7, 795-806.

10. Wang, W.; Li, Z.; Yang, Y.; Guo, Z. Droplet Based Micro Oscillating Flow-Through PCR Chip. In Proceedings of the 17th IEEE International Conference on Micro Electro Mechanical Systems (MEMS), Maastricht, The Netherlands, 25-29 January 2004; pp. 280-283.

11. Qiu, X.; Mauk, M.G.; Chen, D.; Liu, C.; Bau, H.H. A large volume, portable, real-time PCR reactor. Lab Chip 2010, 10, 3170-3177.

12. Hsieh, T.-M.; Luo, C.-H.; Huang, F.-C.; Wang, J.-H.; Chien, L.-J.; Lee, G.-B. Enhancement of thermal uniformity for a microthermal cycler and its application for polymerase chain reaction. Sens. Actuator. B 2008, 130, 848-856.

13. Hsieh, T.-M.; Luo, C.-H.; Wang, J.-H.; Lin, J.-L.; Lien, K.-Y.; Lee, G.-B. Enhancement of thermal uniformity for a microthermal cycler and its application for polymerase chain reaction. Microfluid. Nanofluid. 2009, 6, 797-809.

14. Shen, K.; Chen, X.; Guo, M.; Cheng, J. A microchip-based PCR device using flexible printed circuit technology. Sens. Actuator. B 2005, 105, 251-258.

15. Wang, J.-H.; Chien, L.-J.; Hsieh, T.-M.; Luo, C.-H.; Chou, W.-P.; Chen, P.-H.; Chen, P.-J.; Lee, D.-S.; Lee, G.-B. A miniaturized quantitative polymerase chain reaction system for DNA amplification and detection. Sens. Actuator. B 2009, 141, 329-337.

16. Matsui, T.; Franzke, J.; Manz, A.; Janasek, D. Temperature gradient focusing in a PDMS/glass hybrid microfluidic chip. Electrophoresis 2007, 28, 4606-4611.

17. Ross, D.; Locascio, L.E. Microfluidic temperature gradient focusing. Anal. Chem. 2002, 74, 2556-2564.

18. Yap, Y.F.; Tan, S.H.; Nguyen, N.T.; Murshed, S.M.S.; Wong, T.N.; Yobas, L. Thermally mediated control of liquid microdroplets at a bifurcation. J. Phys. D Appl. Phys. 2009, 42, 1-14.

19. Ting, T.H.; Yap, Y.F.; Nguyen, N.-T.; Wong, T.N.; Chai, J.C.K. Thermally mediated breakup of drops in microchannels. Appl. Phys. Lett. 2006, 89, 234101:1-234101:3.

20. Jiao, Z.; Huang, X.; Nguyen, N.-T.; Abgrall, P. Thermocapillary actuation of droplet in a planar microchannel. Microfluid. Nanofluid. 2008, 5, 205-214.

21. Jiao, Z.; Huang, X.; Nguyen, N.-T. Manipulation of a droplet in a planar channel by periodic thermocapillary actuation. J. Micromech. Microeng. 2008, 18, 045027:1-045027:9.

22. Nguyen, N.T.; Huang, X.Y. Thermocapillary effect of a liquid plug in transient temperature fields. Jpn. J. Appl. Phys. 2005, 44, 1139-1142. 
23. Jiao, Z.J.; Nguyen, N.T.; Huang, X.Y.; Ang, Y.Z. Reciprocating thermocapillary plug motion in an externally heated capillary. Microfluid. Nanofluid. 2007, 3, 39-46.

24. Darhuber, A.A.; Valentino, J.P.; Davis, J.M.; Troian, S.M. Microfluidic actuation by modulation of surface stresses. Appl. Phys.Lett. 2003, 82, 657-659.

25. Darhuber, A.A.; Valentino, J.P. Thermocapillary actuation of droplets on chemically patterned surfaces by programmable microheater arrays. J. Microelectromech. Syst. 2003, 12, 873-879.

26. Darhuber, A.A.; Davis, J.M.; Troian, S.M. Thermocapillary actuation of liquid flow on chemically patterned surfaces. Phys. Fluids 2003, 15, 1295-1304.

27. Darhuber, A.A.; Valentino, J.P; Troian, S.M. Planar digital nanoliter dispensing system based on thermocapillary actuation. Lab Chip 2010, 10, 1061-1071.

28. Selva, B.; Cantat, I.; Jullien, M.-C. Temperature-induced migration of a bubble in a soft microgravity. Phys. Fluids 2011, 23, 052002:1-052002:12.

29. Selva, B.; Marchalot, J.; Jullien, M.-C. An optimized resistor pattern for temperature gradient control in microfluidics. J. Micromech. Microeng. 2009, 19, 065002:1-065002:10.

30. Selva, B; Miralles, V.; Cantat, I.; Jullien, M.-C. Thermocapillary actuation by optimized resistor pattern: bubbles and droplets displacing, switching and trapping. Lab Chip 2010, 10, 1835-1840.

31. Guo, M.T.; Rotem, A.; Heyman, J.A.; Weitz, D.A. Droplet microfluidics for high-throughput biological assays. Lab Chip 2012, 12, 2146-2155.

32. Stroock, A.D.; Dertinger, S.K.W.; Ajdari, A.; Mezic, I.; Stone, H.A.; Whitesides, G.M. Chaotic mixer for microchannels. Science 2002, 295, 647-651.

33. Kim, S.-J.; Wang, F.; Burns, M.A.; Kurabayashi, K. Temperature-programmed natural convection for micromixing and biochemical reaction in a single microfluidic chamber. Anal. Chem. 2009, $81,4510-4516$.

34. Liu, R.H.; Stremler, M.A.; Sharp, K.V.; Olsen, M.G.; Santiago, J.G.; Adrian, R.J.; Aref, H.; Beebe, D.J. Passive mixing in a three-dimensional serpentine microchannel. J. Microelectromech. Syst. 2000, 9, 190-197.

35. Laval, P.; Lisai, N.; Salmon, J.-B.; Joanicot, M. A microfluidic device based on droplet storage for screening solubility diagrams. Lab Chip 2007, 7, 829-834.

36. Velve Casquillas, G.; Fu, C.; Le Berre, M.; Cramer, J.; Meance, S.; Plecis, A.; Baigl, D.; Greffet, J.-J.; Chen, Y.; Piel, M.; Tran, P.T. Fast microfluidic temperature control for high resolution live cell imaging. Lab Chip 2011, 11, 484-489.

37. Velve Casquillas, G.; Costa, J.; Carlier-Grynkorn, F.; Mayeux, A. A fast microfluidic temperature control device for studying microtubule dynamics in fission yeast. Method. Cell Biol. 2010, 97, 185-201.

38. Mao, H.; Yang, T.; Cremer, P.S. A microfluidic device with a linear temperature gradient for parallel and combinatorial measurements. J. Am. Chem. Soc. 2002, 124, 4432-4435.

39. Xia, Y.; Whitesides, G.M. Soft lithography. Angew. Chem. Int. Ed. 1998, 37, 550-575.

40. Maltezos, G.; Johnston, M.; Scherer, A. Thermal management in microfluidics using micro Peltier junctions. Appl. Phys. Lett. 2005, 87, 154105:1-154105:3.

41. De Mello, A.J.; Habgood, M.; Lancaster, N.L.; Welton, T.; Wooton, R.C.R. Precise temperature control in microfluidic devices using Joule heating of ionic liquids. Lab Chip 2004, 4, 417-419. 
42. Mavraki, E.; Moschou, D.; Kokkoris, G.; Vourdas, N.; Chatzandroulis, S.; Tserepi, A. A continuous flow $\mu$ PCR device with integrated microheaters on a flexible polyimide substrate. Procedia Eng. 2011, 25, 1245-1248.

43. Vigolo, D.; Rusconi, R.; Piazza, R.; Stone, H.A. A portable device for temperature control along microchannels. Lab Chip 2010, 10, 795-798.

44. Lao, A.I.K.; Lee, T.M.H.; Hsing, I.-M.; Ip, N.Y. Precise temperature control of microfluidic chamber for gas and liquid phase reactions. Sens. Actuator. A 2000, 84, 11-17.

45. Selva, B.; Mary, P.; Jullien, M.-C. Integration of a uniform and rapid heating source into microfluidic systems. Microfluid. Nanofluid. 2010, 8, 755-765.

46. Ross, D.; Gaitan, M.; Locascio, L.E. Temperature measurement in microfluidic systems using a temperature-dependent fluorescent dye. Anal. Chem. 2001, 73, 4117-4123.

47. Oda, R.P.; Strausbauch, M.A.; Huhmer, A.F.; Borson, N.; Jurrens, S.R.; Craighead, J.; Wettstein, P.J.; Eckloff, B.; Kline, B.; Landers, J.P. Infrared-mediated thermocycling for ultrafast polymerase chain reaction amplification of DNA. Anal. Chem. 1998, 70, 4361-4368.

48. Ferrance, J.P.; Wu, Q.; Giordano, B.; Hernandez, C.; Kwok, Y.; Snow, K.; Thibodeau, S.; Landers, J.P. Developments toward a complete micro-total analysis system for Duchenne muscular dystrophy diagnosis. Anal. Chim. Acta 2003, 500, 223-236.

49. Giordano, B.; Ferrance, J.; Swedberg, S.; Hulhmer, A.; Landers, J. Polymerase chain reaction in polymeric microchips: DNA amplification in less than 240 seconds. Anal. Biochem.2001, 291, 124-132.

50. Ke, C.; Berney, H.; Mathewson, A.; Sheehan, M. Rapid amplification for the detection of Mycobacterium tuberculosis using a non-contact heating method in a silicon microreactor based thermal cycler. Sens. Actuator. B 2001, 102, 308-314.

51. Jagannathan, H.; Yaralioglu, G.; Ergun, A.; Khuri-Yakub, B. Acoustic Heating and Thermometry in Microfluidic Channels. In Proceedings of IEEE the Sixteenth Annual International Conference on Micro Electro Mechanical Systems (MEMS-03 Kyoto), Kyoto, Japan, 19-23 January 2003; pp. 474-477.

52. Kondoh, J.; Shimizu, N.; Matsui, Y.; Sugimoto, M.; Shiokawa, S. Development of temperature-control system for liquid droplet using surface Acoustic wave devices. Sens. Actuator. A 2009, 149, 292-297.

53. Yaralioglu, G. Ultrasonic heating and temperature measurement in microfluidic channels. Sensors Sens. Actuator. A 2011, 170, 1-7.

54. Bykov, Y.V.; Rybakov, K.I.; Semenov, V.E. High-temperature microwave processing of materials. J. Phys. D Appl. Phys. 2001, 34, R55-R75.

55. Shah, J.J.; Geist, J.; Gaitan, M.A. Microwave-induced adjustable nonlinear temperature gradients in microfluidic devices. J. Micromech. Microeng. 2010, 20, 105025:1-105025:8.

56. Kempitiya, A.; Borca-Tasciuc, D.A.; Mohamed, H.S.; Hella, M.M. Localized microwave heating in microwells for parallel DNA amplification applications. Appl. Phys. Lett. 2009, 94, 064106:1-064106:3.

57. Shaw, K.J.; Docker, P.T.; Yelland, J.V.; Dyer, C.E.; Greenman, J.; Greenway, G.M.; Haswell, S.J. Rapid PCR amplification using a microfluidic device with integrated microwave heating and air impingement cooling. Lab Chip 2010, 10, 1725-1728. 
58. Orrling, K.; Nilsson P.; Gullberg, M.; Larhed, M. An efficient method to perform milliliter-scale PCR utilizing highly controlled microwave thermocycling. Chem.Commun. 2004, doi: 10.1039/ B317049G.

59. Shah, J.J.; Sundaresan, S.G.; Geist, J; Reyes, D.R.; Booth, J.C.; Rao, M.V.; Gaitan, M. Microwave dielectric heating of fluids in an integrated microfluidic device. J. Micromech. Microeng. 2007, 17, 2224-2230.

60. Geist, J.J.; Gaitan, M. Microwave power absorption in low-reflectance, complex, lossy transmission lines. J. Res. Natl. Inst. Stand. Technol. 2007, 112, 177-189.

61. Siegel, A.C.; Shevkoplyas, S.S.; Weibel, D.B.; Bruzewicz, D.A.; Martinez, A.W.; Whitesides, G.M. Cofabrication of electromagnets and microfluldic systems in poly(dimethylsiloxane). Angew. Chem. Int. Ed. 2006, 45, 6877-6882.

62. Kappe, C.O.; Dallinger, D. The impact of microwave synthesis on drug discovery. Nat. Rev. Drug Discov. 2006, 5, 51-63.

63. Fermer, C.; Nilsson, P.; Larhed, M. Microwave-assisted high-speed PCR. Eur. J. Pharm. Sci. 2003, 18, 129-132.

64. Issadore, D.; Humphry, K.J.; Brown, K.A.; Sandberg, L.; Weitz, D.A.; Westervelt, R.M. Microwave dielectric heating of drops in microfluidic devices. Lab Chip 2009, 9, 1701-1706.

65. Guijt, R.M.; Dodge, A.; van Dedem, G.W.K.; de Rooij, N.F.; Verpoorte, E. Chemical and physical processes for integrated temperature control in microfluidic devices. Lab Chip 2003, 3, $1-4$.

66. Maltezos, A. Rajagopal, A. Scherer. Evaporative cooling in microfluidic channels. Appl. Phys. Lett. 2006, 89, 074107:1-074107:3.

67. Wu, J.; Cao, W.; Chen, W.; Chang, D.C.; Sheng, P. Polydimethylsiloxane microfluidic chip with integrated microheater and thermal sensor. Biofluidics 2009, 3, 012005:1-012005:7.

68. Robert de Saint Vincent, M.; Wunenburger, R.; Delville, J.-P. Laser switching and sorting for high speed digital microfluidics. Appl. Phys. Lett. 2008, 92, 154105:1-154105:3.

69. Kim, H.; Vishniakou, S.; Faris, G.W. Petri dish PCR: Laser-heated reactions in nanoliter droplet arrays. Lab Chip 2009, 9, 1230-1235.

70. Ohta, A.T.; Jamshidi, A.; Valley, J.K.; Hsu, H.-Y.; Wu, M.C. Optically actuated thermocapillary movement of gas bubbles on an absorbing substrate. Appl. Phys. Lett. 2007, 91, 074103:1-074103:3.

71. Gosse, C.; Bergaud, C.; Löw, P. Molecular probes for thermometry in microfluidic devices. Top. Appl. Phys.2009, 118, 301-341.

72. Liu, R.H.; Yang, J.; Lenigk, R.; Bonanno, J.; Grodzinski, P. Self-contained, fully integrated biochip for sample preparation, polymerase chain reaction amplification, and DNA microarray detection. Anal. Chem. 2004, 76, 1824-1831.

73. Unger, M.A.; Chou, H.-P.; Thorsen, T.; Scherer, A.; Quake, S.R. Monolithic microfabricated valves and pumps by multilayer soft lithography. Science 2000, 288, 113-115.

74. Kartalov, E.P.; Scherer, A.; Quake, S.R.; Taylor, C.R.; Anderson, W.F. Experimentally validated quantitative linear model for the device physics of elastomeric microfluidic valves. J. Appl. Phys. 2007, 101, 064505:1-064505:4. 
75. Pitchaimani, K.; Sapp, B.C.; Winter, A.; Gispanski, A.; Nishida, T.; Fan, Z.H. Manufacturable plastic microfluidic valves using thermal actuation. Lab Chip 2009, 9, 3082-3087.

76. Gu, P.; Liu, K.; Chen, H.; Nishida, T.; Fan, Z.H. Chemical-assisted bonding of thermoplastics/elastomer for fabricating microfluidic valves. Anal. Chem. 2011, 83, 446-452.

77. Deb, K. Multi-Objective Optimization Using Evolutionary Algorithms. Wiley-Blackwell: Hoboken, NJ, USA, 2001; ISBN: 0-471-87339-X.

78. Deb, K.; Pratap, A.; Agarwal, S.; Meyarivan, T. A fast and elitist multiobjective genetic algorithm: NSGA-II. IEEE Trans. Evol. Comput. 2002, 6, 182-197.

79. Vigolo, D.; Rusconi, R.; Stone, H.A.; Piazza R. Thermophoresis: Microfluidics characterization and separation. Soft Matter 2010, 6, 3489-3493.

80. Baroud, C.N.; Delville, J.-P.; Gallaire, F.; Wununburger, R. Thermocapillary valve for droplet production and sorting. Phys. Rev. E 2007, 75, 046302:1-046302:5.

81. Weinert, F.M.; Braun, D. Optically driven fluid along arbitrary microscale patterns using thermoviscous expansion. J. Appl. Phys. 2008, 104, 104701:1-104701:10.

82. Hettiarachchi, K.; Kim, H.; Faris, G.W. Optical manipulation and control of real-time PCR in cell encapsulating microdroplets by IR laser. Microfluid. Nanofluid. 2012, 13, 967-975.

83. Hung, P.J.; Lee, P.J.; Sabounchi, P.; Lin, R; Lee, L.P. Continuous perfusion microfluidic cell culture array for high-throughput cell-based assays. Biotechnol. Bioeng. 2005, 89, 1-8.

84. Mary, P.; Studer, V.; Tabeling, P.; Microfluidic droplet-based liquid-liquid extraction. Anal. Chem. 2008, 80, 2680-2687.

85. Davis, S.H. Thermocapillary instabilities. Annu. Rev. Fluid Mech. 1987, 19, 403-435.

86. Mugele, F.; Baret, J.-C. Electrowetting: From basics to applications. J. Phys. Condens. Matter 2005, 17, 705-774.

87. Moon, H.; Cho, S.K.; Garrell, R.L.; Kim, C.J. Low voltage electrowetting-on-dielectric. J. Appl. Phys. 2002, 92, 4080-4087.

88. Vigolo, D.; Brambilla, G.; Piazza, R. Thermophoresis of microemulsion droplets: Size dependence of the Soret effect. Phys. Rev. E 2007, 75, 040401:1-040401:4.

89. Piazza, R.; Guarino, A. Soret effect in interacting micellar solutions. Phys. Rev. Lett. 2002, 88, 208302:1-208302:8.

90. Cong, H.; Pan, T. Photopatternable conductive PDMS materials for microfabrication. Adv. Funct. Mater. 2008, 18, 1912-1921.

(C) 2013 by the authors; licensee MDPI, Basel, Switzerland. This article is an open access article distributed under the terms and conditions of the Creative Commons Attribution license (http://creativecommons.org/licenses/by/3.0/). 